\title{
Polyethylene glycol treated allografts not tissue matched nor immunosuppressed rapidly repair sciatic nerve gaps, maintain neuromuscular functions, and restore voluntary behaviors in female rats
}

\author{
Michelle Mikesh ${ }^{1}$, Cameron L. Ghergherehchi ${ }^{2}$, Sina Rahesh ${ }^{1}$, Karthik Jagannath ${ }^{1}$, Amir \\ Ali $^{1}$, Dale R. Sengelaub ${ }^{3}$, Richard C. Trevino ${ }^{4}$, David M. Jackson ${ }^{5}$, Haley O. Tucker ${ }^{2}$, and \\ George D. Bittner ${ }^{1}$ \\ ${ }^{1}$ Department of Neuroscience, University of Texas at Austin, Austin, Texas 78712, USA \\ ${ }^{2}$ Department of Molecular Biosciences, University of Texas at Austin, Austin, Texas 78712, USA \\ ${ }^{3}$ Department of Psychological and Brain Sciences, Indiana University, Bloomington, Indiana \\ 47405, USA \\ ${ }^{4}$ Department of Orthopedic Surgery, Wellspan Teaching Hospitals, York, PA, USA
}

${ }^{5}$ Neuraptive Therapeutics, Lafayette CO, USA

\section{Abstract}

\begin{abstract}
Many publications report that ablations of segments of peripheral nerves produce the following unfortunate results: (1) Immediate loss of sensory signaling and motor control, (2) Rapid Wallerian degeneration of severed distal axons within days, (3) Muscle atrophy within weeks, (4) Poor behavioral (functional) recovery after many months, if ever, by slowly-regenerating $(\sim 1 \mathrm{~mm} / \mathrm{d})$ axon outgrowths from surviving proximal nerve stumps. (5) Nerve allografts to repair gap injuries are rejected, often even if tissue matched and immunosuppressed. In contrast, using a female rat sciatic nerve model system, we report that neurorrhaphy of allografts plus a wellspecified-sequence of solutions (one containing polyethylene glycol: PEG) successfully addresses each of these problems by: (a) Re-establishing axonal continuity/signaling within minutes by nonspecifically PEG-fusing (connecting) severed motor and sensory axons across each anastomosis, (b) Preventing Wallerian degeneration by maintaining many distal segments of inappropriatelyreconnected, PEG-fused axons that continuously activate nerve-muscle junctions, (c) Maintaining innervation of muscle fibers that undergo much less atrophy than otherwise-denervated muscle fibers, (d) Inducing remarkable behavioral recovery to near-unoperated levels within days to
\end{abstract}

\footnotetext{
Corresponding Author: George D. Bittner, Department of Neuroscience, The University of Texas at Austin, 2415 Speedway, Austin, TX 78712, Phone: 512-923-3735, Fax: 512-471-9651, bittner@ austin.utexas.edu.

Conflict of Interest Statement

The authors declare no conflicts of interest.

Author Contributions

All authors had full access to all the data in the study and take responsibility for the integrity of the data and accuracy of the data analysis. Conceptualization, MM, CLG, RCT, HOT, GDB; Methodology, MM, CLG, RCT, DRS, DMJ, GDB; Investigation, MM, CLG, AA, SR, KJ; Formal Analysis, MM, CLG, DRS, DMJ, GDB; Writing, MM, CLG, AA, SR, KJ, DRS, RCT, DMJ, HOT, GDB; Supervision, MM, CLG, RCT, DRS, DMJ, GDB; Funding Acquisition, RCT, DRS, DMJ, GDB;
} 
weeks, almost certainly by CNS and PNS plasticities well-beyond what most neuroscientists currently imagine, and (e) Preventing rejection of PEG-fused donor nerve allografts with no tissue matching or immunosuppression. Similar behavioral results are produced by PEG-fused autografts. All results for Negative Control allografts agree with current neuroscience data (1)-(5) given above. Hence, PEG-fusion of allografts for repair of ablated peripheral nerve segments expand on previous observations in single-cut injuries, provoke reconsideration of some current neuroscience dogma and further extend the potential of PEG-fusion in clinical practice.

\section{Graphical abstract}

Behavioral Scores

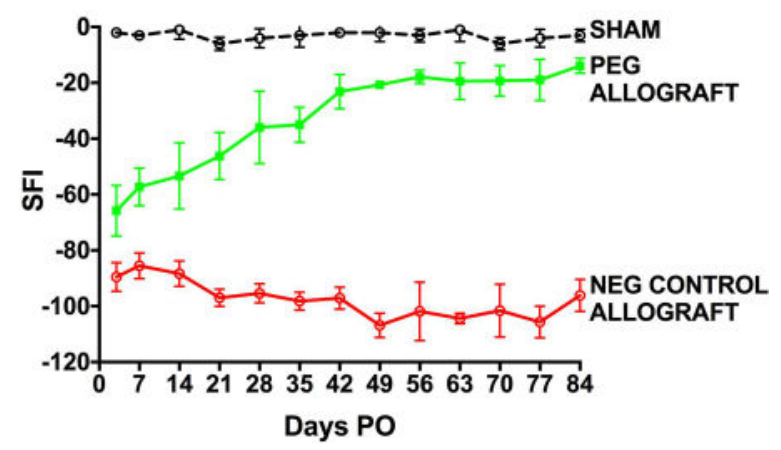

PEG-fused allografts rapidly and dramatically restore behaviors after segmental ablation of rat sciatic nerves.

\section{Key words or phrases}

axotomy; polyethylene glycol; Wallerian degeneration; nerve repair; nerve regeneration; sciatic nerve

\section{Introduction}

Peripheral nerve injuries (PNIs) that result in nerve gap (ablation) defects are common in civilian life and combat trauma (Allan, 2000; Stansbury, 2007; Campbell, 2008). Notably, over 50\% of combat wounds in Operation Iraqi Freedom and Operation Enduring Freedom involved a PNI (Cross et al., 2011; Kang et al., 2011). Patients as well as experimental laboratory animals with nerve gap defects experience: (1) immediate complete loss of sensory and motor functions mediated by the denervated structures, (2) followed by rapid Wallerian degeneration of severed distal axonal segments within only a few days from injury, and (3) slow ( $1 \mathrm{~mm} /$ day) natural regeneration by outgrowths that produce poor (if ever) functional/behavioral recovery. Poor recovery is due in part to non-specific reinnervation and/or to muscle atrophy or deterioration of nerve target structures before reinnervation, if any ever occurs (Brushart, 2011; Kandel et al., 2013).

Current best practices to repair nerve segment ablations in the clinic or in experimental animal models employ: 1) autografts that result in donor site morbidity, 2) donor non- 
allogenic (decellularized) allografts, or 3) synthetic conduits. Each of these structures are microsutured ("neurorrhaphy") to host epineurium and connective tissue at both ends of the gap. These methods are all intended to create a bridge across the gap to guide surviving proximal axons to distal nerve segments. Although axons always rapidly degenerate in distal segments of severed nerves, distal segment Schwann cells continue to be viable and endoneurial sheaths remain intact and combine to act as guides to direct axonal outgrowths from surviving proximal axonal stumps to distal targets (Campbell, 2008; Green and Wolfe, 2011; Bittner et al., 2016a,b; 2017a,b; Bamba et al., 2016a; Mikesh et al., 2018).

These contemporary procedures to repair nerve gaps are problematic in that they do not prevent Wallerian degeneration of distal axonal segments. Therefore, all solely rely on natural regeneration to produce slow and poor recovery of function and/or sensation. Autograft gap repairs also "do harm", i.e., they reduce or eliminate sensation or function subserved by the ablated host donor nerve. Attempts to use viable-cell allografts have not been successful because the grafts are rejected within days due to innate antigenindependent events and $\mathrm{T}$ cell adaptive responses, even when immunosuppression and major histocompatibility complex (MHC) matching are employed (Mackinnon et al., 2001; Riley et al., 2015; Murphy and Weaver, 2016).

Using protocols that employ the membrane fusogen polyethylene glycol (PEG), we have recently demonstrated that viable-cell autografts (Sexton et al., 2012) and allografts (Riley et al., 2015; Bittner et al., 2016a,b; 2017a,b) "PEG-fused" into peripheral nerve gaps dramatically improve the speed and quality of recovery of lost behavioral functions. In contrast to the poor outcomes from conventional repair techniques, animals with $5-10 \mathrm{~mm}$ nerve gaps repaired with viable PEG-fused interposition autografts or (especially) allografts exhibit: (1) excellent restoration of sensory and motor functions beginning as early as three days postoperatively, (2) reduction or prevention of Wallerian degeneration of axons, and (3) reduction or prevention of muscle fiber atrophy or deterioration. However, as reported for single transections in a previous paper (Mikesh et al., 2018), we have not thoroughly examined the structure and/or function of severed donor graft axons or host distal axons, nor neuromuscular junctions (NMJs) and muscle fibers in this model. We have also never quantitatively evaluated the time course of behavioral recovery at various PO times and whether the restored behavior is mediated by: 1) immediate reinnervation by axons that are artificially PEG-fused and then continuously maintained, versus 2) delayed reinnervation by natural regeneration of axons proximal to the lesion site.

In this paper, we confirm and extend our observations described above that female rats with PEG-fused autografts or (especially) allografts exhibit excellent, rapid, and permanent behavioral recovery dramatically better than any other technique to repair peripheral nerves reported to date. We report for the first time that host neuromuscular junctions (NMJs) and muscle fibers are continuously maintained after PEG-fusion, i.e., do not undergo severe atrophy. Most surprisingly, we report that PEG-fused allografts are not rejected even though they are neither tissue matched nor immunosuppressed.

It is logical to conclude that PEG-fused axons, and the target tissues they continuously innervate, are responsible for rapid and dramatically improved behavioral recovery exhibited 
after PEG-fusion. Current Neuroscience dogma holds that regeneration success must rely on the accuracy and specificity of connections made by regenerating axons. In contrast, our remarkable results for PEG-fused allografts occur despite the non-specific connections that are most likely established by PEG-fused host and donor axons and/or perhaps by host axons regenerating by outgrowth. Finally, these results strongly suggest that use of PEGfused allografts could greatly improve the outcomes in clinical repair of peripheral nerve gap injuries.

\section{Methods}

\section{Surgery}

Surgical and other procedures were approved and performed according to standards set by the Institutional Animal Care and Use Committee at the University of Texas at Austin. Animals were maintained on a $12 \mathrm{hr}: 12 \mathrm{hr}$ reverse light:dark cycle and given food and water ad libitum. Surgical and behavioral procedures were performed during the active cycle for these animals. PEG-fusion results for both sexes were very similar for measures such as CAP restoration, dye diffusion, muscle fiber morphology, and SFI recovery (Britt et al., 2010; Bittner et al., 2012, 2016. Riley et al., 2015; Sexton et al., 2012; Rodriguez-Feo et al., 2013; Ghergherehchi et al., 2016). Hence, only females were used in this study to examine effects of PEG-fusion on the same structures because use of both sexes would double animal use.

Female Sprague-Dawley rats weighing 250-300g $(\mathrm{n}=79)$ were anesthetized and maintained with a small inhaled isoflurane anesthetic system (Handlebar Anesthesia, Austin, TX), as previously described for this and other basic surgical procedures in sterile conditions (Mikesh et al., 2018). A 25-35mm incision was made in the skin to expose the sciatic nerve parallel to the biceps femoris muscle. A segment of sciatic nerve was ablated in mid-thigh with fine surgical scissors, leaving an $8-10 \mathrm{~mm}$ gap between cut axonal ends in the proximal and distal stumps. [NB - Intact nerves are under tension so an ablation produces a nerve gap that is several mm longer than the segment removed.]

Host autografts were obtained from distal peroneal nerve segments from the contralateral or ipsilateral hind limb. Two segments of peroneal nerve were used as a cable graft to match nerve diameters. Autografts were of smaller diameter than the host sciatic nerve. Donor allografts were obtained from the left or right sciatic nerve of another rat that was not tissue matched or immune-suppressed in the donor or host. Donor allografts were chosen to approximately match the diameter of the host sciatic nerve at its proximal and distal ends. The autografts or allografts were sized to be $1-3 \mathrm{~mm}$ longer than the nerve gap created by the ablation of a segment of the host sciatic nerve. Autograft and allografts were stored in calcium-free, hypotonic saline (Plasmalyte A) at $2{ }^{\circ} \mathrm{C}$ for $30 \mathrm{~min}-6 \mathrm{hr}$ before use.

The sciatic nerve and ablation site in the thigh of the host rat was flushed with sterile Plasmalyte A and the proximal and distal axonal ends carefully trimmed. For autografts, double cable grafts were inserted into the nerve gap and the cut ends of each cable were carefully trimmed to lie loosely against the trimmed open ends of the host nerve. A similar procedure was followed for the donor allograft that can be regarded as a "single cable". The 
severed, opened, nerve ends were treated with an antioxidant (1\% methylene blue, MB) in sterile distilled water to keep axonal ends open and prevent formation of additional intracellular vesicles. The transected, open ends of the graft and host nerves were closely apposed with microsutures at both ends of the graft. After microsuturing, animals were randomly assigned in PEG-fused or Negative Control groups, as described below.

In PEG-fused animals, after neurorraphy, a sterile hypotonic solution of $50 \% \mathrm{w} / \mathrm{w} 3.35 \mathrm{kDa}$ PEG in distilled water was applied to each repair site for 1-2 minutes to non-specifically repair/join/fuse closely apposed open cut axonal ends. This solution was omitted for Negative Control animals. The microsutured ends of both repair sites in both PEG-fused and Negative Control nerves were then flushed several times with isotonic Lactated Ringers containing calcium to repair axolemmal holes with calcium-induced vesicles. The surgery site was closed with sutures or staples and the animal placed on heating pads to recover.

Electrophysiological analyses-Extracellular compound action potentials (CAPs) were evoked prior to and immediately following surgical procedures in all animals to assess electrophysiological function and continuity (Riley et al., 2015; Mikesh et al., 2018). Stimulating and recording electrodes were placed just proximal and just distal to the autograft or allograft and a CAP was recorded to assess whether axonal continuity was reestablished across the graft. Successful CAP conduction across the lesion site produced twitches of muscles innervated distal to the graft.

Compound muscle action potentials (CMAPs) were evoked prior to and immediately following surgical procedures by stimulating the sciatic nerve in the upper thigh and recording from the tibialis anterior muscle. The stimulating electrode was placed proximally to the autograft or allograft, and a pair of monopolar needle electrodes were placed in the muscle. The stimulus current was carefully controlled to avoid ephaptic stimulation of distal muscle masses (Rupp et al., 2007).

\section{Behavioral analyses}

Animals receiving an autograft or allograft were behaviorally tested using the Sciatic Function Index (SFI) and blindly tested and scored, as described in the previous paper (Mikesh et al., 2018). Animals were handled and trained prior to surgery to acclimate rats to the testing procedures. Animals were tested before any operation to obtain a baseline score, $3 \mathrm{~d}$ PO, then weekly thereafter for up to $84 \mathrm{~d}$ PO. The left (injured) and right (unoperated) hind paws were dipped in blue or red ink, respectively. The rat was placed on a $100 \mathrm{~mm}$-wide wooden board lined with white paper and allowed to return to their home cage. Two trial runs without stopping or hesitation were gathered for each testing day, and an average score calculated for that day. The normal footprint length, experimental footprint length, normal toe spread, experimental toe spread, normal intermediary toe spread and experimental intermediary toe spread were measured for all footprints to generate the SFI score (Carlton and Goldberg, 1986). Some rats would frequently scratch and bite the operated hindlimb, resulting in amputation of one or more toes (autonomy). Such animals were excluded from further behavioral tests to produce reliable SFI scores for all animals included in this study (Varaejao et al., 2001). 
A successful SFI score for animals receiving a PEG-fused allograft was defined as having significantly better $(\mathrm{p}<0.05$ or $\mathrm{p}<0.01)$ SFI score compared with negative control allografts (Ghergherehchi et al., 2016). The mean SFI \pm SD score of all negative control allograft animals was $102.6 \pm 11.4$ at $42 \mathrm{~d} \mathrm{PO}$ and a score of -79.8 and -68.4 at $42 \mathrm{~d} \mathrm{PO}$ indicated successful $\mathrm{p}<0.05$ and $\mathrm{p}<0.01$ recovery, respectively.

Animals receiving an autograft affect the standard calculation for scoring the SFI. Autografts that are taken from the ipsilateral peroneal nerve cause a permanent loss of functions served by the peroneal nerve. These functions (such as ankle dorsiflexion, total toe spread, etc.) are heavily incorporated into the SFI score. This problem (referred as "donor site morbidity") is unavoidable in cases where nerve segments from the host are removed for the repair of nerve gaps. Ipsilateral peroneal nerve harvest also eliminates the chance of PEG-fusing distal peroneal axons, which could contribute to functional recovery. Autografts taken from the contralateral peroneal nerve also present complications with the SFI score. The SFI test is dependent upon the differences in gait between the unoperated and injured hind limbs, and removal of the contralateral peroneal nerve therefore creates a deficit in the "unoperated" hind limb that affects the SFI scores. These limitations cause the SFI test to be less reliable (but not meaningless) for autograft repair compared with other surgical repair techniques.

\section{Immunohistochemical analyses}

Soleus muscles were taken from Unoperated rats $(\mathrm{n}=2)$ and the operated sides of PEG-fused $(n=7)$ or Negative Control $(n=4)$ rats 7, 21, or 42d PO and labelled with antibodies specific to nerves (SV2 and 2H3, DSHB) and conjugated bungarotoxin to label acetylcholine receptors, as previously described (Kang et al., 2007; Mikesh et al., 2018).

\section{Morphological analyses of sciatic axons, nerves, NMJs, and soleus muscle fibers}

After sacrifice by cardiac $\mathrm{KCl}$ or Euthasol, proximal host segments were sampled about $4 \mathrm{~mm}$ proximal to the graft. Donor graft segments were sampled at approximately mid-graft, and distal host segments were sampled about $4 \mathrm{~mm}$ distal to the graft and approximately 10 $12 \mathrm{~mm}$ distal to the proximal transection site. Proximal and distal sections of allograft nerve preparations were sampled 5-8mm from the graft site, comparable to data taken from singly cut nerves in the preceding paper (Mikesh et al., 2018). Much care was taken in this study to ensure that sampling sites were equivalent in all cases relative to suture placement.

Tissue samples were prepared for resin embedding as previously described (Smith et al., 2013; Mikesh et al., 2018). Glass knife thick sections $(0.5 \mu \mathrm{m})$ were stained in toluidine blue and thin sections (65nm) were cut on a diamond knife (DDK) and mounted on formvarcoated Synaptek grids (EMS) prior to viewing in a Technai Spirit electron microscope fitted with an AMT Advantage HR $1 \mathrm{k} \times 1 \mathrm{k}$ digital camera. For axon morphology, electron micrographs were taken at $1700 \times$ and examined and analyzed as previously described (Mikesh et al., 2018). For synaptic ultrastructure, regions with NMJs were thin-sectioned ( $65 \mathrm{~nm})$ and imaged with medium to higher-power TEM $(8,000 \times-26,000 \times)$. Soleus muscle NMJs were assessed using a Zeiss Axiovert 200M. Confocal and other images were captured on a Zeiss LSM 710 with a 40× oil immersion lens and images prepared in Fiji or ImageJ.

J Neurosci Res. Author manuscript; available in PMC 2019 January 01. 


\section{Statistical analyses}

Means, linear regressions and t-test comparisons were calculated using EXCEL. Welch's ttests, a test that allows for heteroscedastic data, was used to compare axonal morphometrics in which $\mathrm{n}=$ the number of animals in a treatment group, $\mathrm{N}=$ the total number of axons in a treatment group, and $\mathrm{N}-2=$ the degrees of freedom. Cohen's $d$ effect size was calculated as the difference between two means divided by a standard deviation for the data (Cohen,1998; Sawilowsky, 2009). Student's two-tailed t-test for homoscedastic data was employed to compare averaged SFI scores for each treatment group on a given post-operative day. Twoway ANOVAs analyzed means and standard errors of SFI scores (Mikesh et al., 2018).

Axon diameters in an undamaged nerve occur in a bimodal, non-normal distribution. Left shifts in distribution are always observed after nerve transections in Negative Control animals. T-tests are the standard test of axon or nerve fiber diameter in peripheral nerve models when comparing conditions or treatments (Ikeda et al., 2012; Cragg and Thomas, 1964; Sherman et.al., 2012) and we have followed this convention.

\section{Animal use for different experimental protocols}

We and others Brushart (2011) assert that the successful repair of any peripheral nerve damage is best measured by behavioral assays such as the SFI. Hence, all animals (79) cited in this study were tested for behavior and initial CAP and CMAP recordings. Different subsets of these animals were also tested for one or more morphological or functional assays. For example, 43 animals were behaviorally (SFI) tested in the present study (11 PEG allografts, 2 PEG autografts, 12 Negative Control allografts, 4 Sham and 14 donor animals). The SFI scores of these animals were compared against the SFI scores of 36 "historical" animals that were single cut nerve repair (14 PEG, 14 Negative Control, 8 cut and not sutured). Of the 43 animals used in this study, 21 animals (3 Unoperated, 9 PEG-fused, 9 Negative Control) were used for analyses of axon and muscle morphology. All animals were tested behaviorally immediately before sacrifice for morphological analyses. Some assays require different fixation or other sampling protocols, and therefore could not be tested using all the assays.

\section{Results}

\section{Terminology}

We use the following terms according to accepted meanings as explicitly defined in Mikesh et al. (2018): Reinnervation, Regeneration, PEG-fusion, Compound action potential (CAP), and Compound muscle action potential (CMAP), nerve axon, and nerve fiber. Unsuccessful PEG-fusions are defined as PEG-fusions in animals that failed to achieve SFI $>-80$ at $42 \mathrm{~d}$ PO, an SFI two standard deviations $(\mathrm{p}<0.05)$ better than recovery observed for Negative Control allografts. The term Autograft refers to viable nerve fibers taken from the host and then inserted and microsutured into a resected portion of a host nerve; an autograft usually produces additional sensory and/or motor deficits to the host, i.e., does harm.

Allografts are viable nerve fibers taken from a donor and then are inserted and microsutured into a resected portion of a host nerve; if the allograft neither induces a severe inflammatory response nor is rejected, an allograft does minimal or no harm to the host. 


\section{Electrophysiological evidence that PEG-fusion produces axonal continuity and reduces or prevents Wallerian degeneration and allograft rejection: CAPs and CMAPs}

As previously described (Bittner et al., 2012, 2016a,b; 2017a,b; Riley et al., 2015; Ghergherehchi et al., 2016; Mikesh et al., 2018), we always confirmed proximal to distal $\mathrm{CAP}$ conduction in intact sciatic nerves prior to lesioning and observe no CAP throughconduction immediately after ablating a nerve segment. Within minutes of PEG-fusing a graft into an ablated segment we always recorded CAP conduction across the inserted autograft or allograft to confirm restoration of axonal continuity (Fig. 1). CAPs also conducted across the PEG-fused allografts and/or autografts when a rat was sacrificed at any time from 7-42d PO (Fig. 1b,c), as well as for allografts at >100d PO (Bittner et al., 2012, 2016a,b; 2017a,b). These CAP recordings demonstrated that PEG-fusion had reestablished morphological and functional continuity of at least some sciatic nerve axons across the autograft and/or allograft from $0->100 \mathrm{~d}$ PO. During these CAP procedures, we also observed twitching of the toes and other muscles innervated distal to a PEG-fused autograft or allograft. These muscle contractions were not observed if the nerve was stimulated, but not PEG-fused. CAPs did not conduct across the transection site immediately after microsuture repair or at 7d PO if the nerve graft was not PEG-fused (Negative Control nerves, Fig. 1a,b). A small CAP was recorded at 42d PO (Fig. 1c). CAPs stimulated proximal to the graft did not produce muscle twitches distal to the graft at any PO time.

CMAPs were recorded from distal muscle fibers (tibialis anterior) after stimulation of the sciatic nerve in intact Unoperated nerves or after PEG-fusion of both ends of an autograft or allograft from 7-42d PO (Fig. 1d-f). CMAPs were not recorded if Negative Control nerves were stimulated proximal to the graft at $0-21 \mathrm{~d}$ PO. CMAPs could be recorded if stimulated distal to the graft at $0 \mathrm{~d} P O$, but not if stimulated $7 \mathrm{~d}-21 \mathrm{~d}$ PO. A small CMAP could sometimes be recorded at $42 \mathrm{~d} \mathrm{PO}$. These observations, along with similar results for CAP recordings (Fig. 1a-c) confirmed immediate and maintained preservation of an ability to evoke voluntary muscle activity after PEG-fusion, and inability to do so at $7-21 \mathrm{~d}$ PO in the absence of PEG-fusion repair. CAPs and CMAPs were not generated by ephaptic current spread after allografts or autografts (also see Mikesh et al., 2018).

\section{Behavioral evidence that PEG-fusion of allografts produces axonal continuity: SFI scores}

As previously described (Bittner et al., 2012, 2016a,b; 2017a,b; Ghergherehchi et al., 2016; Mikesh et al., 2018), we used the SFI as a sensitive test for voluntary behavioral control of the lower limb. The SFI score in this model usually increased over time from surgery because re-innervation of denervated muscles occurs in a proximal-to-distal direction when natural regeneration occurred by outgrowth from the most-proximal transection site. Unoperated or Sham Control animals have SFI scores of \pm 20 . Figure 2a shows the individual SFI scores of PEG-fused allograft, PEG-fused autograft, and Negative Control animals examined at 3, 7, 14, 21, 35 and 42d PO. Although no recovery was present in Negative Control animals, significant functional (behavioral) recovery occurred by $42 \mathrm{~d} P O$ for all successfully PEG-fused animals; in fact, some rats with successfully PEG-fused allografts recovered to near baseline levels within 14 days. 
Figure $2 b$ shows the average \pm SE SFI scores for each of these experimental groups.

Negative control animals show an immediate loss of sciatic nerve mediated functions, and these functions were not significantly restored by $42 \mathrm{~d}$ PO. Since the autograft was taken ipsilateral or contralateral to the host limb receiving the autograft, SFI recovery of autografts was more difficult to assess compared to Unoperated controls or Negative Controls (see Methods). PEG-fused allografts typically exhibited significant behavioral recovery beginning at $7 \mathrm{~d}-14 \mathrm{~d} P O(\mathrm{p}<0.01)$, and recovery to near baseline levels at $21-42 \mathrm{~d} P O(\mathrm{p}<$ $0.001)$. One PEG-fused allograft (G-28, see Table 2) showed significant ( $p<0.01)$ SFI recovery to -66 compared to Negative Controls ( -100 to -125$)$, but less than other PEGfused allografts $(-22$ to +25$)$.

Unoperated animals were able to lift their heel and spread their toes during normal gait. Negative control animals immediately lost the ability to lift their heels during gait (most often seen as dragging of the foot during walking), and were no longer able to properly spread their toes for a normal gait pattern. In contrast, PEG-fused animals regained the ability to spread toes and lift their heel almost completely within $42 \mathrm{~d} \mathrm{PO}$ and walked with a near-normal gait. Individual SFI scores of animals tested for 42d PO (Fig. 2a,b) showed that PEG-fused animals had variable SFI scores at all PO times; that is, some animals recovered almost all functions rapidly, while other animals slowly recovered these functions over time. Negative Control animals exhibited much less variation in SFI scores from $3 \mathrm{~d}-42 \mathrm{~d}$ PO, and rarely showed any gain of behavioral functions at any time (Fig. 2a,b).

Figure 2c shows the combined average \pm SE SFI scores for all animals tested for $42 \mathrm{~d}$ or $84 \mathrm{~d}$ PO for Sham Control, PEG-fused allografts, PEG-fused autografts, and Negative Control animals that used the same PEG-fusion protocols described in this and the preceding paper (Mikesh et al., 2018). Note that there were no significant differences between our current data and comparable historical values for any surgical procedure. Negative controls allografts and single cut (no microsutures) protocols showed no significant recovery at any time up to $84 \mathrm{~d} \mathrm{PO}$. Cut+microsuture animals recovered some functions by $63-84 \mathrm{~d} \mathrm{PO}$ ( $\mathrm{p}<$ 0.05). PEG-fused single cuts recovered within $14 \mathrm{~d} P O(\mathrm{p}<0.05)$, exhibited increase recovery by $21-28 \mathrm{~d}$ PO ( $\mathrm{p}<0.01)$, and plateaued around $35 \mathrm{~d}$ PO ( $\mathrm{p}<0.001)$. PEG-fused allografts showed significant recovery by $3-7 \mathrm{~d} P O(\mathrm{p}<0.01)$, and recovered to near baseline by $35 \mathrm{~d} \mathrm{PO}(\mathrm{p}<0.001)$.

\section{Morphological evidence that PEG-fusion produces and maintains axonal continuity and reduces or prevents Wallerian degeneration and allograft rejection: gross structure of sciatic nerves}

As reported for singly-transected sciatic nerves, we examined morphological features of nerve and muscle. Unoperated and 0-42d PO PEG-fused and Negative Control sciatic nerves were examined for their gross anatomical features with a dissecting microscope (Fig. $3)$.

Figure 3a shows that Unoperated nerves glisten silver-white and often have little change in diameter from the upper thigh to a bifurcation near the knee joint. Several small blood vessels were typically visible along the surface of the nerve that did not adhere to surrounding tissues. PEG-fused grafts at Od PO (Fig. 3b: autograft, Fig. 3c: allograft) also 
had a silver-white appearance and constant diameter from proximal host neve, across the graft and into the distal host tissue. PEG-fused allografts at 42d PO (Fig. 3d) maintained a silver-white appearance and constant diameter from proximal host neve, across the graft and into the distal host tissue with no or slight adhesions to surrounding tissue, consistent with tissue that was viable and not immune-rejected. Negative Control allografts (Fig. 3e) at 42d PO usually had a reddish-yellow color. Sciatic nerves in Negative Control allograft were often swollen to twice the size of the host nerve and were covered with material that was difficult to dissect from surrounding tissues and sometimes obscured one or both of the suture sites, observations consistent with nonviable tissue that was immune-rejected.

\section{Morphological evidence that PEG-fusion produces and maintains axonal continuity and reduces or prevents Wallerian degeneration and allograft rejection: TEM}

Type of data shown in Figs 4-9 and Tables 2-3-Tissue structures and more detailed axon morphology were examined with TEM transverse section images at 1.7 - 6kx (Figs. 45,7-9), NMJs at $8-20 \mathrm{kx}$, and filamentous ultrastructure, mitochondria, and myelin layers of individual axons were observed at $26-43 \mathrm{kx}$ at $80 \mathrm{kv}$. Figures 4 and 5 show representative low-power cross-section electron micrographs from Unoperated sciatic nerves or from proximal, graft or distal portions of PEG-fused or Negative Control sciatic nerves at 7d, 21d, or $42 \mathrm{~d}$ PO.

Nerve axon diameters are expressed as frequency distribution histograms in Figure 7 and nerve fiber diameters as box plots in Figure 8, gathered from images like those shown in Figures 4 and 5. These and other morphological data for sciatic nerves and the soleus muscle they innervate are summarized in Table 2 and statistical analyses summarized in Table 3.

Morphology of Unoperated myelinated axons and fibers-Myelinated axons in Unoperated nerves had electron-lucid, organized axoplasm with surrounded by electrondense rings of myelin (Figs. 4-6, Mikesh et al., 2018). Blood vessels were open with few non-red blood cell profiles in the lumen and had a well-defined endothelium (not shown). Axonal diameter in the proximal segment averaged $3.95 \pm 1.74 \mu \mathrm{m}$ with a $0.63 \pm 0.05 \mathrm{~g}$ ratio (551 axons, $\mathrm{n}=3$ ) and distal axons averaged $3.82 \pm 1.64 \mu \mathrm{m}$ with a $0.61 \pm 0.06 \mathrm{~g}$ ratio (522 axons, $\mathrm{n}=3$ ), all non-significant differences (Tables 2 and 3). Nerve fiber diameter (total diameter of myelinated axons) averaged $6.4 \pm 2.7 \mu \mathrm{m} \mathrm{SD}$. The average diameter of the ten largest nerve fibers $\left(\mathrm{MAX}_{10}\right)$ was $12.0 \pm 0.6 \mu \mathrm{m}$ proximally and $11.6 \pm 0.3 \mu \mathrm{m}$ distally, a nonsignificant difference. The $\mathrm{g}$ ratio versus axon diameter plots for proximal versus distal portions of the sciatic nerve in the thigh did not differ significantly. Given the similarity of these values, we combined Unoperated proximal and distal data for Figures 7-9 and present the same data for Unoperated sciatic nerves in all panels.

Morphology of PEG-fused vs Negative Control myelinated axons and fibersCompared to Unoperated nerves, the morphology of proximal nerve segments for PEGfused or Negative Control nerves exhibited little change relative to those within the graft or distal segments. As described below, the morphology of PEG-fused nerves showed some change within the allograft or distal segments at $7 d-41 d$ PO. In contrast, the morphology of 
Negative Control nerves underwent rather drastic changes within the allograft and distal segments at 7d-42d PO (Figs. 4-9, Tables 2-3).

Morphology at 7d PO-Myelinated axons in PEG-fused donor allografts were intact with organized axoplasm, and myelin sheaths had slightly larger diameters in the graft and distal segment at 7d PO (Fig. 4, Table 2). Blood vessels were open with few non-red blood cell profiles in the lumen (arrowhead, Fig.6a) and had a well-defined endothelium. Axons in a PEG-fused allograft had an average diameter of $3.88 \pm 1.41 \mu \mathrm{m}$ and a $\mathrm{g}$ ratio of $0.67 \pm 0.05$ within the graft, and $4.44 \pm 1.74 \mu \mathrm{m}$ and $0.69 \pm 0.03$ in the distal host segment. Nerve fiber diameters in the graft averaged $5.8 \mu \mathrm{m}$ with $\mathrm{MAX}_{10}=9.5 \mu \mathrm{m}$ and those in the distal segment averaged $6.4 \mu \mathrm{m}$ with $\mathrm{MAX}_{10}=11.0 \mu \mathrm{m}$. These values were very similar to Unoperated values (see above, Figs. 4-9, and Tables 2 and 3). In contrast, at 7d PO, Negative Control allografts had no intact myelinated axons or organized myelin compared to Unoperated or PEG-fused nerves in the donor graft or distal host segments. That is, Negative Control axons in the allograft and distal to the allograft had very disorganized ultrastructure such as a lack of ordered axoplasmic organelles and filaments, and much electron dense material of unknown origin, comparable to that described for singly-cut nerves at 7d PO (Mikesh et al., 2018). Myelin sheaths were collapsed and disintegrating. Therefore, no measurements for axons or myelin sheaths could be made for Negative Control animals at 7d PO (Table 2; Figs. 4-9). Blood vessels in Negative Controls were often obstructed with many cell types and endothelial cells were abnormal (Fig. 6b).

Morphology at 21d PO-PEG-fused allografts continued to have many myelinated axons with normal axoplasmic ultrastructure (e.g., tubulin, neurofilament arrangement) and myelin periodicity proximal to, within, and distal to the allograft at $7 \mathrm{~d}$ PO. Most myelinated axons were single (unbundled) and within their own basal lamina sheath, and their myelin appeared generally normal. Few non-neuronal cells infiltrated the nerve space (Fig. 6c). Axonal diameters in PEG-fused animals decreased within the graft and distally (Figs. 4, 7-9, Table 2) compared to Unoperated nerves. Axons within allografts had average diameters of $2.62 \pm 1.11 \mu \mathrm{m}$ with a $0.72 \pm 0.07 \mathrm{~g}$ ratio. Axons distal to the graft had average diameters of $2.01 \pm 0.99 \mu \mathrm{m}$ with a $0.62 \pm 0.08 \mathrm{~g}$ ratio. Average nerve fiber diameter in the graft and distal segments was $3.6 \mu \mathrm{m}$ and $3.2 \mu \mathrm{m}, \mathrm{MAX}_{10}=7.4$ and $6.9 \mu \mathrm{m}$ respectively. All these values were significantly smaller than those of Unoperated nerves but significantly larger than those of Negative Control nerves.

In contrast, only one of two 21d PO Negative Control nerves had any myelinated axons in the distal host segment, and these axons were very small. These small axons had normalappearing ultrastructure. Densely packed lymphocyte-type cells and debris occupied much of the nerve space (Fig. 6d). Those few axons that were observed within the graft had average diameters of $2.24 \pm 0.74 \mu \mathrm{m}$ with a $0.81 \pm 0.06 \mathrm{~g}$ ratio. The average diameter of the few distal axons were also very small $(1.35 \pm 0.22 \mu \mathrm{m})$ and the $g$ ratio high $(0.78 \pm 0.07)$. Negative Control nerve fibers had an average diameter $4.1 \mu \mathrm{m}$ with $\mathrm{MAX}_{10}=6.6 \mu \mathrm{m}$ in grafts, and $2.4 \mu \mathrm{m}$ with $\mathrm{MAX}_{10}=2.6 \mu \mathrm{m}$ in the distal host segment.

Morphology at 42d PO-The normal structure of axons and myelin in all host and donor regions of PEG-fused allografts was maintained at 42d PO (Figs. 5-9, Table 2). Specifically, 
axons in successfully-PEG-fused allografts $(\mathrm{n}=6)$ averaged $2.82 \pm 1.48 \mu \mathrm{m}$ in diameter with $0.65 \pm 0.07 \mathrm{~g}$ ratio and distal axons averaged $2.85 \pm 1.71 \mu \mathrm{m}$ in diameter with a $0.67 \pm 0.08 \mathrm{~g}$ ratio. Nerve fibers at $42 \mathrm{~d} P O$ in the allograft and distal segments averaged $4.2 \mu \mathrm{m}$ and $4.2 \mu \mathrm{m}$ with $\mathrm{MAX}_{10}=11.4 \mu \mathrm{m}$ and $11.9 \mu \mathrm{m}$, respectively. These values were slightly but significantly smaller $(\mathrm{p}<0.05)$ than those of Unoperated nerves but significantly larger $(\mathrm{p}<0.001)$ than those of Negative Control nerves. At 42d PO, Negative Control allografts had fewer and smaller diameter axons in the allograft and even fewer in the distal host segment, most of which did not innervate muscle fibers (Table 2). Axons within the allograft averaged $2.07 \pm 0.83 \mu \mathrm{m}$ with a $0.72 \pm 0.10 \mathrm{~g}$ ratio, and axons in the distal host segment had $1.59 \pm 0.62 \mu \mathrm{m}$ with $0.63 \pm 0.10 \mathrm{~g}$ ratios. Nerve fibers in the Negative Control graft and distal segments averaged 3.6, and 2.6 $\mu \mathrm{m}$ with $\mathrm{MAX}_{10}=7.1$, and $4.7 \mu \mathrm{m}$ respectively.

Morphology of myelinated nerves in less successful and unsuccessfully PEGfused allografts at 42d PO-As given in Table 2, one PEG-fused allograft produced very significant behavioral recovery of the SFI to -66 (animal G-28), but not to the near unoperated range of \pm 20 , as did 5 other allograft repairs at $42 \mathrm{~d}$ PO. Morphometric measures for animal G-28 were intermediate between Unoperated and Negative Control nerves except for muscle fiber diameters that were very close to those observed for Negative Control animals. Omitting animal G-28 from PEG-fused morphometric measures in Table 2 (or Figs. 7-9) produced very little change in any measure (see Table 2, 42d PO PEG-Fused, $n=5$ vs $\mathrm{n}=6$ averages). In contrast, one animal (G-20) with a PEG-fused allograft produced no SFI behavioral recovery at any PO time. The axonal morphology and SFI-assessed behavior of this Unsuccessful PEG-fusion closely resembled those of Negative Control nerves (Fig. 5, Table 2).

Summary of differences-Table 3 shows the results of Welch's T and Cohen's $d$ size effect tests between Unoperated (proximal and distal when comparing to graft, or distal only), PEG, and Negative Control axons in the graft and distal segments. At all times and in all segments, axons in nerves receiving PEG-fused allografts were significantly larger than those in nerves with Negative Control allografts. At most times and in most segments, diameter and $\mathrm{g}$ ratio were significantly different between Unoperated, PEG, and Negative Control axons. Negative Control repaired nerves exhibit uniformly very large effects on axon diameter compared to nerves of Unoperated animals, while PEG-fused axons had smaller effects at all times in all segments. Most notably, PEG-fused axon diameters at $7 \mathrm{~d}$ PO were very similar to those of Unoperated axons in the graft segment, and only moderately different from those in the distal segment $(d=0.37)$. Regeneration by outgrowth from surviving proximal host nerves at $21 \mathrm{~d}-42 \mathrm{~d} \mathrm{PO}$ might be in part responsible for a population of smaller axons seen in PEG-fused allografts.

\section{Morphological evidence that PEG-fusion produces axon continuity and reduces or prevents Wallerian degeneration and allograft rejection: nerve composition}

Figure 9 shows the composition according to PO time and treatment type per $10,000 \mu \mathrm{m}^{2}$ of Unoperated, PEG-fused and Negative Control nerves proximal to, within, and distal to the allograft. As previously described (Mikesh et al., 2018), TEM images were selected at random, and analyzed using Image $\mathbf{J}$ to calculate areas occupied by organized axonal and 
myelin structures and areas occupied by debris, e.g., osmophilic structures such as dissociated myelin, condensed axon material, or material engulfed within macrophages. Interspace areas were defined as electron lucid open space or normally observed nonneuronal cells and calculated as the total area minus the sum of axon, myelin, and debris. Each type of area was converted to a percentage of the total area measured (Mikesh et al., 2018).

Proximal Unoperated nerve segments $(\mathrm{n}=3)$ had areas of $28 \pm 1 \%$ axon, $45 \pm 1 \%$ myelin,, $0 \pm 0 \%$ debris, and $27 \pm 0.5 \%$ interspace area with a density of $210 \pm 24$ axons per $10,000 \mu \mathrm{m}^{2}$. Distal unoperated nerve segments were composed of $27 \pm 1 \%, 47 \pm 3 \%, 26 \pm 3 \%, 0 \pm 0.5 \%$, and $26 \pm 3 \%$, areas occupied by axon, myelin, interspace and debris respectively with a density of $191 \pm 18$ axons per $10,000 \mu^{2}$. Differences between Unoperated proximal and distal segments were not significant and these data were combined for the Unoperated values shown in Figure 9.

At 7d PO (Fig 9a), the donor graft areas in a PEG-fused allograft nerve were $21 \%$ axon, $30 \%$ myelin, $0 \%$ debris and $49 \%$ interspace with 154 axons per $10,000 \mu \mathrm{m}^{2}$. In the host segment distal to the graft, the sciatic nerve areas were $25 \%$ axon, $32 \%$ myelin, $0 \%$ debris, and $43 \%$ interspace with 147 axons per 10,000 $\mu^{2}$. In contrast, at $7 \mathrm{~d} \mathrm{PO}$, a Negative Control allograft nerve in the donor graft segment areas were $<1 \%$ axon, $<1 \%$ myelin, $43 \%$ debris, and $56 \%$ interspace. In the distal host segment, there were no axon or myelin areas and the nerve was composed of $29 \%$ debris and $71 \%$ interspace. That is, the $7 \mathrm{~d} \mathrm{NC}$ had essentially no axon or myelin areas and much more debris and interspace areas compared to Unoperated and PEG-fused nerves that were rather similar.

At 21d PO (Fig 9b) in PEG-fused nerves, areas of proximal host segments were $21 \%$ axon, $42 \%$ myelin, $1 \%$ debris, and $36 \%$ interspace with 192 axons per $10,000 \mu^{2}{ }^{2}$. A Negative control proximal segment was similarly composed of $11 \%$ axon, $20 \%$ myelin, $5 \%$ debris, and $65 \%$ interspace with 150 axons per 10,000 $\mathrm{m}^{2}$. At 21d PO, PEG-fused donor graft segments were composed of $15 \%$ axon, $13 \%$ myelin, $2 \%$ debris, and $70 \%$ interspace with $307 \pm 23$ axons per $10,000 \mu^{2}$, and distal host segments were composed of $14 \%$ axon, $22 \%$ myelin, $1 \%$ debris, and $63 \%$ interspace with a density of $448 \pm 38$ axons per $10,000 \mu \mathrm{m}^{2}$. That is, at $21 \mathrm{~d}$ PO, PEG-fused allografts showed some increases in interspace and decrease in axon diameter and myelin thickness. In contrast, Negative Control donor grafts and host distal nerves had almost no axonal and myelin areas and greatly increased areas of debris and interspace. That is, Negative Control donor grafts were composed of $1 \%$ axon, $1 \%$ myelin, $24 \%$ debris, and $74 \%$ interspace with a density of $18 \pm 14$ axons per $10,000 \mu \mathrm{m}^{2}$. The distal host nerve segments were composed of $0 \%$ axon, $0 \%$ myelin, $11 \%$ debris, and $89 \%$ interspace with $6 \pm 3$ axons per $10,000 \mu \mathrm{m}^{2}$.

At 42d PO, PEG-fused allograft regions showed some increases in interspace, but all regions more closely resembled that of Unoperated nerves than Negative Control nerves (Fig. 9). For example, at 42d PO (Fig 9c), PEG-fused allograft nerve areas in proximal host segments were $22 \%$ axon, $28 \%$ myelin, $1 \%$ debris, and $48 \%$ interspace with a density of $211 \pm 31$ axons per $10,000 \mathrm{\mu m}^{2}$. PEG-fused donor graft areas were $16 \%$ axon, $24 \%$ myelin, $10 \%$ debris, and $50 \%$ interspace with a density of $280 \pm 40$ axons per $10,000 \mu \mathrm{m}^{2}$. Distal host 
segments were $13 \%$ axon, $17 \%$ myelin, $11 \%$ debris, and $59 \%$ interspace with $210 \pm 55$ axons per $10,000 \mu \mathrm{m}^{2}$. Negative Control proximal areas were $16 \%$ axon, $27 \%$ myelin, $6 \%$ debris, and $51 \%$ interspace with a density of $175 \pm 42$ axons per $10,000 \mu \mathrm{m}^{2}$, i.e., slightly less axon and myelin areas compared to Unoperated and PEG-fused animals. However, Negative Control donor graft areas were $6 \%$ axon, $5 \%$ myelin, $6 \%$ debris, and $81 \%$ interspace with a density of $160 \pm 110$ axons per $10,000 \mu \mathrm{m}^{2}$ (all of small diameter). Distal host areas were $3 \%$ axon, $5 \%$ myelin, $6 \%$ debris and $86 \%$ interspace with a density of $151 \pm 111$ axons per $10,000 \mu \mathrm{m}^{2}$. The area composition of an unsuccessful PEG-fused axon resembled that of the Negative Control axons. That is, the unsuccessful proximal host segment was composed of $16 \%$ axon, $22 \%$ myelin, $10 \%$ debris, and $52 \%$ interspace with a density of 126 axons per $10,000 \mu \mathrm{m}^{2}$.

The unsuccessful donor graft segment was composed of 5\% axon, $2 \%$ myelin, $1 \%$ debris, and $92 \%$ interspace with a density of 107 axons per $10,000 \mu \mathrm{m}^{2}$. The distal host segment was $4 \%$ axon, $5 \%$ myelin, $5 \%$ debris and $86 \%$ interspace with 159 axons per $10,000 \mu \mathrm{m}^{2}$. The unsuccessful PEG-fused host segments were much more like Negative Control nerves than successfully PEG-fused nerves.

At all PO times, the nerve composition of PEG-fused allografts distal to the most-proximal transection site more closely resembled the composition of Unoperated intact nerves than did Negative Control nerves. The difference was most striking at $7 \mathrm{~d}$ PO with a PEG-fused nerve having near-normal axon and myelin areas with no debris and Negative Control nerves having no axon and myelin areas with much debris. This difference was maintained to a lesser extent at 21d PO. At 42d PO, PEG-fused nerves did have some debris and Negative Control nerves did have some axon and myelin areas due to small diameter, lightly myelinated axons regenerating by outgrowth from surviving proximal stumps (see previous section, Figs. 4-8, and Table 2).

\section{Morphological evidence that PEG-fusion produces axon continuity and reduces or prevents Wallerian degeneration and allograft rejection: NMJs and muscle fibers}

To demonstrate that axons distal to the PEG-fusion repair site maintained synaptic contact, we labelled neuromuscular junctions (NMJs) in soleus muscles distal to Unoperated, PEGfused and Negative Control sciatic allografts with antibodies specific to neurofilament and synaptic vesicle protein-2 (2H3/SV2) and acetylcholine receptors (AChRs) with fluorescent bungarotoxin. Figure 10 shows representative confocal images of NMJs distal to Unoperated, PEG-fused, and Negative Control allografts at 7d, 21d, and 42d PO, and TEM images of synaptic clefts $42 \mathrm{~d}$ PO. In healthy, undamaged adult muscle tissue, each muscle fiber was innervated by a single axon (Fig. 10a,d,g). The axon (arrowheads) approached the $\mathrm{AChR}$, branched, and mirrored the shape of the receptor with a high degree of fidelity (Fig. $10 \mathrm{~g}$ ). At the ultrastructural level, nerve terminals (NT) in the synaptic cleft were directly opposite secondary folds (Fig. 10j, asterisks), the muscle was well-organized with normal mitochondria (M), and nerve terminals were capped by terminal Schwann cell (TSC) processes. Normal, undamaged muscle fibers were innervated at a rate of $>99 \%$, as observed in other never-denervated muscles in our lab and in other studies (Gutmann and Sanders, 1943; Miledi and Slater, 1968; Sanes and Lichtman, 1999). Distal to a PEG-fused allograft 
7d PO, 50\% (66/131) of muscle fiber AChRs were innervated. In most, though not all muscle fibers, nerve terminals mirrored the shape of the AChR (Fig. 10b). Some sprouting of nerve terminals was seen (not shown). In PEG-fused animals at 21d PO, soleus muscles had 92\% AChR innervation (111/121). AChRs were often multiply innervated (not shown). Most nerves maintained near perfect register with the AChR (Fig. 10e), but some variability was noted (receptor fragmentation, partial innervation, terminal sprouts). At 42d PO, soleus muscles distal to PEG fused allografts were 99\% innervated (111/112). Many NMJs were multiply innervated (double arrowhead, Fig. 10h) or had retraction bulbs near innervated NMJs (not shown). The ultrastructure of nerve terminals of PEG-fused 42d PO appeared normal, were capped by terminal Schwann cells, and apposed most secondary folds of the AChR.

In Negative Control muscles, no nerves were labeled in muscles at $7 \mathrm{~d}$ or $21 \mathrm{~d}$ PO. At $42 \mathrm{~d}$ PO, a few small diameter axons were near AChRs, but no receptors were occupied. Labelled axons parfocal and in contact with AChRs were considered to innervate the receptor. According to this definition, soleus muscles distal to a Negative Control allograft were $9 \%$ innervated (11/129) at $42 \mathrm{~d}$ PO.

\section{Correlations between various morphometric and behavioral (SFI) measures in animals with Unoperated, PEG-fused or Negative Control sciatic nerves as given in Table 2-For Unoperated rats that had SFI scores of -15 to +3 , we observed no significant correlations between SFI scores and various neuromuscular morphometric measures such as sciatic nerve axonal or fiber diameters, axonal density, \% NMJ innervation, or muscle fiber area (Fig. 11a-f, filled circles). There were also no significant correlations at $42 \mathrm{~d}$ PO between SFI scores and various neuromuscular morphometric measures proximal, within or distal to the allograft for Negative Control rats, all of which had SFI scores of -125 to -100 (Fig. 11a-f, $\times$ symbols). Although the variation in SFI scores was much greater for Negative Control rats than for Unoperated rats, these results are to be expected because no negative control rat showed any significant return of behavioral function from $3 \mathrm{~d}-42 \mathrm{~d} \mathrm{PO}$ and all Unoperated rats had behavioral function within the normal range of SFI scores. Furthermore, there were no significant correlations at $42 \mathrm{~d}$ PO between SFI scores and various neuromuscular morphometric measures proximal to the allograft for all PEG-fused rats having SFI scores of -102 to +25 (Fig. 11a, open circles, grey triangle) or for all rats having successfully PEG-fused nerves with SFI scores of -66 to +25 (Fig. 11a, open circles).}

In contrast at $42 \mathrm{~d} P O$, all PEG-fused rats and/or rats with successfully PEG-fused allografts had significant correlations between SFI scores and several neuromuscular morphometric measures (Fig. 11b-f, open circles, grey-filled triangle), such as average axonal diameter in the graft (Fig. 11b, p= 0.0056, 0.023), average distal axonal diameter (Fig. 11c: $\mathrm{p}=0.060$, 0.0071 ), percent graft nerve fiber area (Fig. 11d: $p=0.12,0.0097$ ), percent distal nerve fiber area (Fig. 11e: $p=0.024,0.0097$ ) and distal axonal density (Fig. 10f, $p=0.035,0.041$ ). Other correlations (not shown) were not significant such as SFI versus nerve density within or distal to the allograft. Note that the unsuccessfully PEG-fused nerve (animal G20, Table 2) with an SFI score of -102 exhibited neuromuscular measures comparable to those obtained for Negative Control nerves having similar SFI scores. 


\section{Discussion}

\section{Summary of Results of allograft or autograft repair of ablated segments of rat sciatic nerves}

Unoperated nerves have few obvious changes in morphology in proximal versus distal regions of the thigh in rats. Experimental animals having 6-8mm ablations subsequently repaired by PEG-fused allografts (or autografts) remarkably exhibit:

1. Axolemmal and axoplasmic continuity that is rapidly (within minutes) restored as assessed by conduction of CAPs across the two PEG-fused lesion sites (Fig. 1a-c), and by CMAPs stimulated proximal to the allograft and recorded from muscle groups distal to the allograft (Fig. 1d-f) or autograft (data not shown). [Other publications document diffusion of intraaxonal dyes across PEG-fused allografts (Riley et al, 2015; Bittner et al, 2016a,b)].

2. Continuous maintenance from $0-42 \mathrm{~d} P O$ of distal segments of host and donor allograft axons, i.e., Wallerian degeneration is reduced or prevented, as assessed by gross anatomical inspection of allografts (Fig. 3) or autografts (data not shown) and TEM in allografts of axonal cross sections (Figs. 4-9, Table 2) of myelinated axons (Figs. 4-9) and of the entire sciatic nerve (Fig. 9, Table 2). At all PO times and in all observed segments of the nerve, axons in successfully PEG-fused nerves display normal ultrastructure with respect to myelin periodicity, tubulin and neurofilament arrangement, and mitochondria. Successfully PEG-fused nerves show some increases in interspace area and changes in axonal diameter and myelination, but overall gross morphology remains consistent from the proximal host through the graft and into the distal host region.

3. Continuous maintenance of NMJs in allografts, as measured by CMAPs (Fig. 1d-f), confocal immunohistochemistry (Fig. 10) and TEM observations (Fig. 10) and counts of innervated muscle fibers (Table 2).

4. Continuous maintenance (e.g., minimal atrophy compared to Unoperated animals) of muscle fibers in allografts compared with Negative Controls, as assessed by TEM (Figs. 5,10; Table 2).

5. Behavioral function that is restored more rapidly and to levels significantly better than Negative Controls in allografts and autografts (Fig. 2). In allografts, the restoration of the SFI approaches or equals that of Unoperated animals (Fig. 2, Table 2).

[N.b. The success of nerve repair after a PNI is always defined by behavioral measures such as the SFI and not axon counts or any other morphological or electrophysiological measure, as Brushart (2011) has emphasized. For allografts, PEG-fusion non-selectively fuses proximal to distal axons at both repair sites. That is, PEG-fusion repair does not fuse motor to motor axons nor sensory to sensory axons, much less specific motor to specific motor axons. For allografts, there is no axon-to-axon specificity between donor and host nerves.

J Neurosci Res. Author manuscript; available in PMC 2019 January 01. 
Nevertheless, behavioral recovery is superior for PEG-fused allografts compared to all other PEG-fusion protocols (see Fig. 2).]

6. Non-rejection (immune-acceptance) of PEG-fused allografts despite no tissuematching or immunosuppression as assessed by photon microscopy, TEM, histological or immuno-histochemical methods (Figs. 3-10, Table 2). The lack of rejection is remarkable, unexpected, and contrary to current immunological and neuroscientific dogma. That is, unlike CNS neuronal tissue, mammalian peripheral nerves are in a non-immuno-privileged environment similar to other commonly transplanted organs such as hearts, livers and kidneys that require tissue-matching and immunosuppression to avoid rejection.

In strong contrast, Negative Control allografts that are not PEG-fused do not exhibit any of the phenomena 1-6 above for 7-21d PO and show no significant SFI recovery at $42 \mathrm{~d}$ PO. The occasional slight functional recovery at $42 \mathrm{~d}$ PO is almost-certainly due to smalldiameter myelinated axons regenerating by outgrowth from the severed proximal stumps Figs. 4-10), of which a few reach denervated target organs at 42d PO (Table 2). Muscle fibers are small and often bear central nuclei and are rarely innervated by $42 \mathrm{~d}$ PO.

\section{Comparisons of our results to previous studies of allograft PEG-fusion}

The results described herein confirm and greatly extend our previous data describing allograft repair sciatic nerve ablations that leave an $8-10 \mathrm{~mm}$ gap in the mid-thigh of rats of (Riley et al., 2015). This previous study assessed recovery by SFI scores and CAPs at Od PO and by intra-axonal dye diffusion at $\mathrm{Od}$ PO. Survival of myelinated axons was assessed by histological staining of paraffin-embedded at $7-14 \mathrm{~d} \mathrm{PO}$ and by toluidine blue staining of myelinated axons at $42 \mathrm{~d}$ PO. That is, the current study extensively examines axons, NMJs and muscle fibers at various PO times from $7-42 \mathrm{~d}$ PO by CAP conduction, CMAP production, TEM morphometry of axons, NMJs and muscle fibers, and immuno-staining of NMJs. This study extensively documents prevention of Wallerian degeneration of a significant proportion of axons within a PEG-fused nerve. We report that PEG-fused nerves morphologically have many larger diameter axons with thick wrappings of intact myelin and functionally generate CAPs and CMAPs at all PO times. Postsynaptic muscle fibers have relatively normal-appearing NMJs (Fig. 9 and Tables 2,3) with features consistent with synaptic competition and significantly less atrophy than muscle fibers in Negative Control animals (Smith et al., 2013; Mikesh et al., 2018). These maintained axons, myelin, and NMJs are PEG-fused nerve fibers because regenerating axons do not grow across the nerve gap for at least $21 \mathrm{~d}$ PO and very few soleus muscle fibers distal to the allograft are innervated at $42 \mathrm{~d} \mathrm{PO}$.

It is worth noting that axons maintained by PEG-fusion did undergo some changes from their original intact state, as assessed in Unoperated nerves. For example, CAPs and CMAPs recorded at $7 \mathrm{~d}-42 \mathrm{~d} P O$ have slower conduction velocities and smaller amplitudes than CAPs or CMAPs from intact nerves. While myelinated axons and innervated NMJs are present at all sampling times from $7 \mathrm{~d}-42 \mathrm{~d}$ PO, axonal and fiber diameter and myelin in PEG-fused nerves are smaller than in Unoperated nerves (Table 2, Figs. 5-7). The number of smaller diameter axons increases in PEG-fused nerves at 21d and 42d PO (Table 2, Figs. 4-8) are 
likely due to some regeneration by outgrowth from the proximal stump. Average crosssectional area of muscle fibers is significantly greater than their areas in Negative Control animals but significantly less than their cross-sectional areas in Unoperated animals.

In contrast, Negative Control animals have no surviving axons in the graft or distal nerve at $7 \mathrm{~d}$, very few (if any) regenerating axons in the graft or distal host nerve at $21 \mathrm{~d} P O$, no detectably innervated NMJ's in soleus muscle fibers from 7-21d PO, and very few detectable NMJs at $42 \mathrm{~d}$ PO. That is, all severed distal host and donor axons undergo Wallerian degeneration by $7 \mathrm{~d}$ PO as reported for singly-transected nerves (Mikesh et al., 2018). Negative Control allograft-repaired nerves have much more debris than Unoperated or PEG-fused nerves at 7d PO [Unoperated nerves essentially had no debris]. That debris is largely removed by $21 \mathrm{~d}$ PO and remains at about the same level at $42 \mathrm{~d} \mathrm{PO}$. Many more small-diameter myelinated nerve fibers regenerating by outgrowth are seen distally at $42 \mathrm{~d}$ PO, of which a few reinnervate distal muscle masses. Negative Control allograft animals rarely demonstrate any significant behavioral recovery by $42 \mathrm{~d}$ PO. These data are consistent with other reports that distal axonal segments undergo obligatory Wallerian degeneration within 7 days after an ablation-type injury and then slowly regrow at $\sim 1 \mathrm{~mm} /$ day to sometimes poorly re-innervate denervated muscles after several months (Gutmann and Sanders, 1943; Gutmann and Young, 1944; Reynolds and Woolf, 1992; Gramsbergen et al., 2000; Ijkema-Paassen et al., 2002; Brushart, 2011).

\section{PEG-fusion repair of singly-cut vs ablated segments of rat sciatic nerves}

Table 4 compares morphometric data at three different PO times for PEG-fusion and Negative Control neurorrhaphy repair of single-cut versus ablation-allograft lesions. These summary results are taken from Table 2 giving single cut data of Mikesh et al. (2018) and Table 2 giving allograft data of the Results section of the study described herein. At any given PO time, the PEG-fused single cut and allograft data are very similar and the Negative Control single cut versus allograft data are very similar. In contrast, at any given PO time, the PEG-fused single cut and allograft data versus the Negative Control single cut and allograft data are very different. PEG-fused results are highlighted in yellow, allograft results given in black font and single cut results in red font (in parentheses).

Table 4 as a summary of our data in these two papers consistently demonstrates that: (1) Animals with PEG-fused single cuts or allografts recover lost functions more rapidly and completely compared to negative controls as assayed by the SFI in column 2. (2) Animals with PEG-fused sciatic nerves maintain axons, NMJs, and muscle fibers at all PO times, while Negative Control preparations undergo Wallerian degeneration, denervation, and muscle fiber atrophy, as assessed by morphometric measures in columns 3-13. (3) Negative Control sciatic nerves have smaller diameter axons that regenerate into the distal stump by 21d PO and a few regenerating axons reach denervated muscles by $42 \mathrm{~d}$ PO. (4) Measures of $\mathrm{g}$ ratios and axon numbers were more variable and did not correlate well $(\mathrm{p}>0.05)$ with SFI scores, as reported for regeneration by axonal outgrowth from proximal stumps (Brushart, 2011). 


\section{Some hypotheses that might explain results from these PEG-fused sciatic nerves}

As hypothesized for singly transected sciatic nerves (Mikesh et al., 2018), the observations for PEG-fused allografts described above might be explained by hypothesizing that PEGfusion rapidly re-establishes and maintains continuity for many axons across the donor allograft and distal host nerve and as a consequence, distal target NMJs become and remain functionally reinnervated instead of undergoing Wallerian degeneration, but not appropriately activated for 14-42d PO. In this hypothesis, non-specifically PEG-fused axons undergo central and/or peripheral reorganization that would explain the time lag between immediate neuronal reinnervation and extensive behavioral restoration within days to weeks. Slow natural regeneration of axons not PEG-fused (which could also undergo central reorganization) contributes little, if any, to behavioral recovery by $42 \mathrm{~d}$ PO and perhaps little thereafter as recovery typically plateaus by $42 \mathrm{~d} \mathrm{PO}$. Results reported here and in a previous paper on PEG-fusion of singly transected sciatic nerves (Mikesh et al., 2018) consistently support this hypothesis. Consistent with this hypothesis, PEG-fused axons in allografted nerves conduct action potentials, release transmitter to cause distal muscles to contract and nerve terminals occupy AChRs at all PO times. Also, NMJ innervation is maintained as evidenced by CMAPs at all PO times and improving SFI scores that require an increasing recruitment of more appropriately coordinated muscle contractions. Morphometric analyses of PEG-fused, allografted animals using TEM of axonal, myelin, NMJ and muscle structure at $42 \mathrm{~d} \mathrm{PO}$ are similar to Unoperated nerves.

As an alternate hypothesis previously suggested for singly transected nerves (Mikesh et al., 2018), it is possible that PEG-fusion of axons, which is rapid but non-specific, could induce nerve terminals to become non-functional, retract and regrow, and again be functional within weeks. If this were to be true, CMAPs at 7d PO would not be recordable in PEG-fused animals nor muscle fiber innervation be apparent (Fig. 1, Tables 2,3; Fig. 9). In another scenario, both PEG-fused and non-PEG-fused axons should rapidly (Wallerian) degenerate by $7 \mathrm{~d} \mathrm{PO}$ and enhanced debris clearance and axonal outgrowths from the proximal stump would be needed to re-innervate denervated muscle masses within several weeks. This hypothesis is even more unlikely for allografts than it is for singly transected nerves. That is, PEG-fused nerves in allografts would have to clear almost all debris by $7 \mathrm{~d} P O$ and accelerate nerve outgrowth to about $60 \mathrm{~mm} /$ day for soleus NMJs to display $50 \%$ nerve re-innervation at 7d PO (Tables 2,3; Fig. 8). However, our PEG-fused allograft data are consistent with the hypothesis that the rate $(\sim 1 \mathrm{~mm} / \mathrm{d})$, extent, and morphology of regenerating axons in PEGfused nerves is very similar to that observed for our Negative Control nerves and that reported for other preparations (Brushart, 2011). These are important data because others (Robinson and Madison, 2016) have assumed that all axons that innervate denervated muscles at 42 or more days are axons that have "naturally" regenerated by outgrowths from surviving proximal stumps after they attempt PEG-fusions for the femoral branch of the sciatic nerve. Our data in this and the preceding paper strongly suggest that most, or all, of the axons these authors dye-labeled are PEG-fused axons that are maintained from 0d to $>42 \mathrm{~d}$ PO, not axons that have regenerated by outgrowth.

\section{Comparisons with autografts, synthetic materials or allografts to repair nerve gap PNIs-When neurorrhaphy is not an acceptable option because tension-free, end-to-}


end repair of a longer nerve gap cannot be performed to appose proximal and distal severed nerve ends, cable autografts are generally considered to be the next best option. However, cable autografts themselves produce additional sensory and/or motor deficits and rarely produce good functional recoveries for long gaps in major, mixed motor/sensory nerve ablations, e,g., sciatic nerve ablations in mid-thigh. Furthermore, no other material or approach has shown greater ability to enhance functional recovery than cable autografts. Examples of alternate techniques include conduits made of collagen, porous or smoothwalled poly-L-lactic acid, poly(glycerol sebacate), and polylactic-co-glycolic acid. Other examples would include vein grafts, small intestinal submucosa seeded with Schwann cells, cytokines, other trophic substances and/or cells such as mesenchymal stem cells (for reviews, see Arslantunali et al., 2014; Aikeremujiang and Ao, 2015). Although results of morphometric and electrophysiological analyses of repairs made using these appliances may approach or exceed those reported for cable autografts, functional tests when performed produce uniformly poor results, even when compared to autografts. PEG-fusion of synthetic or acellular conduits having no viable axons would also not be expected to enhance regenerative success. Finally, neurorrhaphy of allografts that are tissue-matched and immunosuppressed have also been used with very limited success to repair nerve gaps (Mackinnon et al., 2001), i.e., tissue-matched and immunosuppressed Negative Controls.

\section{Non-rejection of PEG-fused allografts}

Data reported herein provide substantial morphological and functional evidence that PEGfused allografts are not rejected even though they are neither tissue-matched nor immunosuppressed. That is, many nerve axons, Schwann cells, and other tissues in PEGfused allografts remain viable and exhibit relatively little degenerative changes, but are nonfunctional and exhibit extensive degradation in Negative Control allografts. Relatively few immune cells having morphological characteristics of macrophages or T-cells are seen in PEG-fused allografts, but many are seen in Negative Control allografts.

It is not yet known why PEG-fused allografts are not rejected. One possible mechanism is restoration of axonal transport across a fusion site may allow host MHC proteins to be introduced into the axolemmas of donor allografts. PEG-fused allografts might then avoid being recognized as non-self and escape immune surveillance and targeting. Irrespective of mechanism, the lack of rejection of PEG-fused allografts is not due to some neuroprotective effects that PEG might also have in dilute solutions or when chemically-bound in hydrogels (Kwon et al., 2009; Bittner et al., 2016a; 2017a,b).

\section{Extensive CNS and PNS plasticity explains dramatic behavioral recovery after PEG-fusions}

PEG-fusion of allografts to produce immediate repair of some axons in the donor graft and host distal nerve does not prevent natural regeneration of non-fused axons by outgrowths from surviving host stumps proximal to the allograft. However, our data show that such outgrowth adds little or nothing to the recovery obtained by surviving PEG-fused axons in allografts at $42 \mathrm{~d}$ PO. Hence, PEG-fusion of an ablation and neurorrhaphy of an allograft insertion must produce its dramatic functional/behavioral recovery by activating peripheral and CNS plasticities, quite possibly to a much greater extent than most neuroscientists currently believe to be possible (Riley et al., 2015; Bittner et al., 2016a,b; Bittner et al., 
2017a,b). As noted in our accompanying paper (Mikesh et al., 2018) and demonstrated in a previous publication (Bittner et al., 2017b), this systems level adaptation may include a profound reorganization that restores a normal pattern of behavior ('multiple realizability'; Krakauer, 2017). Trophic substances in the periphery, spinal cord and higher brain centers may help direct such re-organizations.

Clinically, extensive CNS plasticities may be occurring in nerve transfers (neurotization) in humans (refs). For a nerve lesion with a very poor prognosis, an "expendable" motor or sensory nerve can be redirected to a more important target. For example, neurorrhaphy of an ulnar nerve laceration above the elbow never produces functioning hand intrinsic musculature. However, a distal nerve transfer neurorrhaphy of the anterior interosseous nerve to the motor portion of the ulnar nerve near the wrist provides some functioning hand intrinsic musculature after many months. The axonal mismatches in allograft PEG-fusion may produce even greater CNS and PNS re-organization than current nerve transfers, leading to a more rapid, more complete recovery.

\section{Conclusions}

The principal aim of this paper and the accompanying paper (Mikesh et al., 2018) was to describe anatomic changes within nerves and target tissues in parallel to behavioral assessments to begin to provide a more complete understanding at systems, cellular and molecular levels of these remarkable PEG-fusion results. Our results strongly suggest that PEG-fusion maintains axonal continuity, innervation of motor synapses, prevents extensive atrophy of muscle fibers distal to an extensive nerve gap, and enables recovery. These results are consistent with the hypothesis that PEG-fusion immediately re-establishes and then maintains axonal continuity for many axons and most NMJs remain functionally reinnervated, but many not appropriately activated until $14-42 \mathrm{~d} P O$, and irrespective of the non-selectivity of the axonal connections established by the technique.

The results of PEG-fused allografts contradict much current Neuroscience dogma asserting that: (1) Distal stumps of severed host axons or donor allograft axons must undergo Wallerian degeneration within days, (2) Reinnervation after nerve ablations can exclusively occur by outgrowths regenerating from severed host proximal stumps that must appropriately reinnervate denervated host target tissues, and (3) Allografts, like other tissues in a non-protected immune environment, must be quickly rejected in the absence of tissuematching and immunosuppression. In contrast, our results show that: (1) Distal stumps of PEG-fused severed allograft donor or host axons survive indefinitely, i.e., never undergo Wallerian degeneration, (2) PEG-fusion induces rapid reinnervation by connecting axons in proximal and distal stumps to non-selectively reinnervate denervated target tissues, (3) Dramatic behavioral recovery by PEG-fused allografts must be due to inappropriate connections that undergo extensive peripheral and/or CNS plasticities that support functional recovery, and (4) PEG-fused allografts are not rejected despite the lack of tissue matching or immunosuppression. Our results also strongly suggest that voluntary behavior is the best measure of recovery success after nerve transection followed by any experimental procedure include PEG-fusion. Morphological measures such as the ratio of myelin thickness to axon diameter ( $r=0.53$, de Medinaceli, 1995) or axon numbers (Mackinnon, 1991; Brushart, 2011) 
do not correlate well with behavioral recovery, with the possible exception of axon or nerve fiber diameter (Mikesh et al., 2018).

Finally, ablations producing long gaps of larger-diameter mixed sensory-motor peripheral nerves in more proximal limbs always results in extensive long-term disability in experimental animal models or in the clinic with any technique published to date. PEGfusion has obvious potential to greatly benefit patients with such injuries by dramatically increasing the speed and magnitude of functional recovery. To date, limited clinical case studies $(\mathrm{n}=5)$ strongly suggest that PEG-fusion indeed produces very favorable outcomes for single transection injuries (Bamba et al., 2016b; Trevino, personal communications). Use of PEG-fusion in conjunction with neurorraphy for single-cut and ablation injuries has the potential to greatly contribute to clinical outcomes. It is possible that protocols to PEG-fuse ablated peripheral nerves may produce equal or better results without immunosuppression or tissue matching, i.e., tissue banks for peripheral nerves might one day be possible. If further confirmed, these PEG-fusion results for single transections and/or complete ablations in experimental animal models and/or clinical case studies may foreshadow the most significant advance to repair PNIs since the first attempts made over a century ago (Howell and Huber, 1893; Raffe, 1985).

\section{Acknowledgments}

\section{Other Acknowledgements}

We wish to thank the Microscopy and Imaging Facility of the Center for Biomedical Research Support at The University of Texas at Austin for access and assistance with confocal and electron microscopy, and the University of Texas Department of Statistics and Data Sciences for consulting services.

Support or grant information: Supported by grants from the Lone Star Paralysis Foundation to GDB, NIH grant R01 NS081063 to GDB and Lone Star Paralysis grant to RCT.

\section{References}

Aikeremujiang M, Ao Q. Past, present, and future of nerve conduits in the treatment of peripheral nerve injury. Biomed Res Int. 2015; 2015:1-6. DOI: 10.1155/2015/237507

Allan CH. Functional results of primary nerve repair. Hand Clin. 2000; 16:67-72. [PubMed: 10696577]

Arslantunali D, Dursun T, Yucel D, Hasirici N, Hasirici V. Peripheral nerve conduits: technology update. Med Devices (Auckl). 2014; 7:405-424. DOI: 10.2147/MDER.S59124 [PubMed: 25489251]

Bamba R, Riley C, Kelm ND, Does MD, Dortch RD, Thayer WP. A novel technique using hydrophilic polymers to promote axonal fusion. Neural Regen Res. 2016a; 11(4):525-528. DOI: 10.4103/1673-5374.180724 [PubMed: 27212898]

Bamba R, Waitayaawinyu T, Nookala R, Riley DC, Boyer RB, Sexton KW, Boonyasirrikool C, Niempoog S, Kelm ND, Does MD, Dortch RD, Shack RB, Thayer WP. A novel therapy to promote axonal fusion in human digital nerves. J Trauma Acute Care Surg. 2016b; 81:S177-S183. DOI: 10.1097/TA.0000000000001203 [PubMed: 27768666]

Bittner GD, Keating CP, Kane JR, Britt JM, Spaeth CS, Fan JD, Zuzek A, Wilcott RW, Thayer WP, Winograd JM, Gonzalez-Lima F, Schallert T. Rapid, effective, and long-lasting behavioral recovery produced by microsutures, methylene blue, and polyethylene glycol after completely cutting rat sciatic nerves. J Neurosci Res. 2012; 90:967-980. DOI: 10.1002/jnr.23023 [PubMed: 22302646] 
Bittner GD, Mikesh M, Ghergherehchi CL. PEG-fusion retards Wallerian degeneration and rapidly restores behaviors lost after nerve severance. Neural Regen Res. 2016a; 11:217-219. DOI: 10.4103/1673-5374.177716 [PubMed: 27073362]

Bittner GD, Sengelaub DL, Ghergherehchi CL. Conundrums and confusions regarding how PEGfusion produces excellent behavioral recovery after peripheral nerve injuries. Neural Regen Res. 2017a; : 12.doi: 10.4103/1

Bittner GD, Sengelaub DR, Trevino RC, Ghergherehchi CL, Mikesh M. Robinson and Madison have published no data on whether polyethylene glycol fusion repair prevents reinnervation accuracy in rat peripheral nerve. J Neurosci Res. 2017b; 95:863-866. DOI: 10.1002/jnr.23849 [PubMed: 27514994]

Bittner GD, Sengelaub DR, Trevino RC, Peduzzi JD, Mikesh M, Ghergherehchi CL, Schallert T, Thayer WP. The curious ability of polyethylene glycol fusion technologies to restore lost behaviors after nerve severance. J Neurosci Res. 2016b; 94:207-230. DOI: 10.1002/jnr.23685 [PubMed: 26525605]

Britt JM, Kane JR, Spaeth CS, Zuzek A, Robinson GL, Gbanaglo MY, Estler CJ, Boydston EA, Schallert T, Bittner GD. Polyethylene glycol rapidly restores axonal integrity and improves the rate of motor behavior recovery after sciatic nerve crush injury. J Neurophysiol. 2010; 104:695-703. DOI: 10.1152/jn.01051.2009 [PubMed: 20445038]

Brushart, TM. Nerve Repair. NY: Oxford University Press; 2011.

Campbell WW. Evaluation and management of peripheral nerve injury. Clin Neurophysiol. 2008; 119:1951-1965. DOI: 10.1016/j.clinph.2008.03.018 [PubMed: 18482862]

Carlton JM, Goldberg NH. Quantitating integrated muscle function following reinnervation. Surg Forum. 1986; 37:611-612.

Cohen, J. Statistical power analysis for the behavioral sciences. NJ: Lawrence Erlbaum Associates; 1998.

Cragg BG, Thomas PK. The conduction velocity of regenerated peripheral nerve fibres. J Physiol. 1964; 171(1):164-75. DOI: 10.1113/jphysiol.1964.sp007369 [PubMed: 14170140]

Cross JD, Ficke JR, Hsu JR, Masini BD, Wenke JC. Battlefield orthopaedic injuries cause the majority of long-term disabilities. J Am Acad Orthop Surg. 2011; 19(Suppl 1):S1-S7. [PubMed: 21304041]

de Medinaceli L. Interpreting nerve morphometry data after experimental traumatic lesions. J Neuroscience Methods. 1995; 58:29-37. DOI: 10.1016/0165-0270(94)00156-B

Ghergherehchi CL, Bittner GD, Hastings RL, Mikesh M, Riley DC, Trevino RC, Schallert T, Thayer WP, Sunkesula SR, Ha TA, Munoz N, Pyarali M, Bansal A, Poon AD, Mazal AT, Smith TA, Wong NS, Dunne PJ. Effects of extracellular calcium and surgical techniques on restoration of axonal continuity by polyethylene glycol fusion following complete cut or crush severance of rat sciatic nerves. J Neurosci Res. 2016; 94:231-245. DOI: 10.1002/jnr.23704 [PubMed: 26728662]

Gramsbergen A, Ijkema-Paassen J, Meek MF. Sciatic Nerve Transection in the Adult Rat: Abnormal EMG Patterns during Locomotion by Aberrant Innervation of Hindleg Muscles. Exp Neurol. 2000; 161:183-193. DOI: 10.1006/exnr.1999.7233 [PubMed: 10683284]

Green, DP., Wolfe, SW. Green's operative hand surgery. 6. Philadelphia: Elsevier/Churchill Livingstone; 2011.

Gutmann E, Sanders FK. Recovery of fibre numbers and diameters in the regeneration of peripheral nerves. J Phys. 1943; 101:489-518.

Gutmann E, Young JZ. The re-innervation of muscle after various periods of atrophy. J Anat. 1944; 78:15-43. [PubMed: 17104938]

Howell WH, Huber GC. A physiological, histological, and clinical study of the degeneration and regeneration in peripheral nerve fibres after severance of their connections with the nerve centres. $\mathrm{J}$ Physiol. 1893; 14:1-51. DOI: 10.1113/jphysiol.1893.sp000439

Ijkema-Paassen J, Meek MF, Gramsbergen A. Reinnervation of muscles after transection of the sciatic nerve in adult rats. Muscle Nerve. 2002; 25:891-897. DOI: 10.1002/mus.10130 [PubMed: 12115979]

Ikeda M, Oka Y. The relationship between nerve conduction velocity and fiber morphology during peripheral nerve regeneration. Brain and Behavior. 2012; 2:382-390. DOI: 10.1002/brb3.61 [PubMed: 22950042] 
Kandel, ER., Schwartz, JH., Jessell, TM., Siegelbaum, SA., Hudspeth, AJ. Principles of neural science. 5. New York: Elsevier; 2013.

Kang H, Tian L, Son YJ, Zuo Y, Procaccino D, Love F, Hayworth C, Trachtenberg J, Mikesh M, Sutton L, Ponomareva O, Mignone J, Enikolopov G, Rimer M, Thompson W. Regulation of the intermediate filament protein nestin at rodent neuromuscular junctions by innervation and activity. J Neurosci. 2007; 27:5948-5957. DOI: 0.1523/JNEUROSCI.0621-07.2007. [PubMed: 17537965]

Kang JR, Zamorano DP, Gupta R. Limb salvage with major nerve injury: current management and future directions. J Am Acad Orthop Surg. 2011; 19(Suppl 1):S28-S34. [PubMed: 21304044]

Krakauer JW, Ghazanfar AA, Gomez-Marin A, MacIver MA, Poeppel D. Neuroscience Needs Behavior: Correcting a Reductionist Bias. Neuron. 2017; 93:480-490. DOI: 10.1016/j.neuron. 2016.12.041 [PubMed: 28182904]

Kwon BK, Roy J, Lee JH, Okon E, Zhang H, Marx JC, Kindy MS. Magnesium chloride in a polyethylene glycol formulation as a neuro-protective therapy for acute spinal cord injury: preclinical refinement and optimization. J Neurotrauma. 2009; 26:1379-1393. [PubMed: 19317592]

Mackinnon SE, Dellon AL, O'Brien JP. Changes in nerve fiber numbers distal to a nerve repair in the rat sciatic nerve model. Muscle Nerve. 1991; 14:1116-22. [PubMed: 1745287]

Mackinnon SE, Doolabh VB, Novak CB, Trulock EP. Clinical outcome following nerve allograft transplantation. Plast Reconstr Surg. 2001; 107:1419-29. [PubMed: 11335811]

Marzullo TC, Britt JS, Stavisky R, Bittner GD. Cooling enhances in vitro survival and fusion-repair of severed axons taken from the peripheral and central nervous system of rats. Neuroscience Letters. 2001; 327:9-12.

Mikesh M, Ghergherehchi CL, Hastings RL, Ali A, Rahesh S, Jagannath K, Sengelaub DR, Trevino $\mathrm{RC}$, Jackson DM, Bittner GD. Polyethylene glycol solutions rapidly restore and maintain axonal continuity, neuromuscular structures and behaviors lost after sciatic nerve transections in female rats. J Neurosci Res. 2018 In press.

Miledi R, Slater CR. Electrophysiology and Electron-Microscopy of Rat Neuromuscular Junctions After Nerve Degeneration. Proc R Soc B Biol Sci. 1968; 169:289-306. DOI: 10.1098/rspb. 1968.0012 [PubMed: 4384567]

Murphy, K., Weaver, C. Janeway’s Immunobiology. 9. New York, NY: Garland Science/Taylor \& Francis Group, LLC; 2016.

Raffe, MR. Principles of peripheral nerve repair. In: Newton, CD., Nunamaker, DM., editors. Textbook of Small Animal Orthopedics. Ithaca, NY: International Veterinary Information Service; 1985.

Reynolds ML, Woolf CJ. Terminal Schwann cells elaborate extensive processes following denervation of the motor endplate. J Neurocytol. 1992; 21:50-66. [PubMed: 1346630]

Riley DC, Bittner GD, Mikesh M, Cardwell NL, Pollins AC, Ghergherehchi CL, Bhupanapadu Sunkesula SR, Ha TN, Hall BT, Poon AD, Pyarali M, Boyer RB, Mazal AT, Munoz N, Trevino RC, Schallert T, Thayer WP. Polyethylene glycol-fused allografts produce rapid behavioral recovery after ablation of sciatic nerve segments. J Neurosci Res. 2015; 93:572-583. DOI: 10.1002/jnr.23514 [PubMed: 25425242]

Robinson GA, Madison RD. Polyethylene glycol fusion repair prevents reinnervation accuracy in rat peripheral nerve. J Neurosci Res. 2016; 94:636-644. DOI: 10.1002/jnr.23734 [PubMed: 26994857]

Rupp A, Dornseifer U, Fischer A, Schmahl W, Rodenacker K, Jutting U, Gais P, Biemer E, Papadopulos N, Matiasek K. Electrophysiologic assessment of sciatic nerve regeneration in the rat: surrounding limb muscles feature strongly in recordings from the gastrocnemius muscle. $\mathrm{J}$ Neurosci Methods. 2007; 166:266-77. DOI: 10.1016/j.jneumeth.2007.07.015 [PubMed: 17854904]

Sanes JR, Lichtman JW. Development of the vertebrate neuromuscular junction. Annu Rev Neurosci. 1999; 22:389-442. DOI: 10.1146/annurev.neuro.22.1.389 [PubMed: 10202544]

Sawilowsky SS. New Effect Size Rules of Thumb. J Modern Applied Statistical Methods. 2009; 8:26.doi: $10.22237 /$ jmasm/1257035100 
Schallert T, Bittner GD. Polyethylene glycol rapidly restores axonal integrity and improves the rate of motor behavior recovery after sciatic nerve crush injury. J Neurophysiol. 2010; 104:695-703. DOI: 10.1152/jn.01051.2009 [PubMed: 20445038]

Sea T, Ballinger ML, Bittner GD. Cooling of peripheral myelinated axons retards Wallerian degeneration. Exp. Neurol. 1995; 133:85-95. DOI: 10.1006/exnr.1995.1010 [PubMed: 7601266]

Sexton KW, Pollins AC, Cardwell NL, Del Corral GA, Bittner GD, Shack RB, Nanney LB, Thayer WP. Hydrophilic polymers enhance early functional outcomes after nerve autografting. J Surg Res. 2012; 177:392-400. [PubMed: 22521220]

Sherman DL, Krols M, Wu LM, Grove M, Nave KA, Gangloff YG, Brophy PJ. Arrest of myelination and reduced axon growth when Schwann cells lack mTOR. J. Neurosci. 2012; 32(5):1817-25. DOI: 10.1523/JNEUROSCI.4814-11.2012 [PubMed: 22302821]

Smith IW, Mikesh M, Lee Yi, Thompson WJ. Terminal Schwann Cells Participate in the Competition Underlying Neuromuscular Synapse Elimination. J Neurosci. 2013; 33:17724-17736. DOI: 10.1523/JNEUROSCI.3339-13.2013 [PubMed: 24198364]

Stansbury LG, Branstetter JG, Lalliss SJ. Amputation in military trauma surgery. J Trauma. 2007; 63:940-944. DOI: 10.1097/TA.0b013e31814934d8 [PubMed: 18090027]

Sunio A, Bittner GD. Cyclosporin retards the Wallerian degeneration of peripheral mammalian axons. Exp. Neurol. 1997; 146:46-56. DOI: 10.1006/exnr.1997.6484 [PubMed: 9225737]

Rodriguez-Feo CL, Sexton KW, Boyer RB, Pollins AC, Cardwell NL, Nanney LB, Shack RB, Mikesh MA, McGill CH, Driscoll CW, Bittner GD, Thayer WP. Blocking the P2×7 receptor improves outcomes after axonal fusion. J Surg Res. 2013; 184:705-713. DOI: 10.1016/j.jss.2013.04.082 [PubMed: 23731685]

Varejão AS, Meek MF, Ferreira AJ, Patrício JA, Cabrita AM. Functional evaluation of peripheral nerve regeneration in the rat: walking track analysis. J Neurosci Methods. 2001; 108(1):1-9. DOI:

10.1016/S0165-0270(01)00378-8 [PubMed: 11459612] 


\section{Significance Statement}

PEG-fused allografts that are neither immunosuppressed nor tissue matched rapidly and dramatically restore behavioral functions after ablating segments of rat sciatic nerves. PEG-fused allografts prevent Wallerian degeneration of many axons within a fused nerve and result in rapid and superior restoration of function despite inducing motor and sensory axonal connections that are almost-certainly very nonspecific with respect to the originally innervated targets. These results contradict contemporary Neuroscience dogma, imply central and peripheral plasticities much greater than currently believed possible, and may foreshadow the most significant advance in the history of clinical repair of peripheral nerve injuries. 

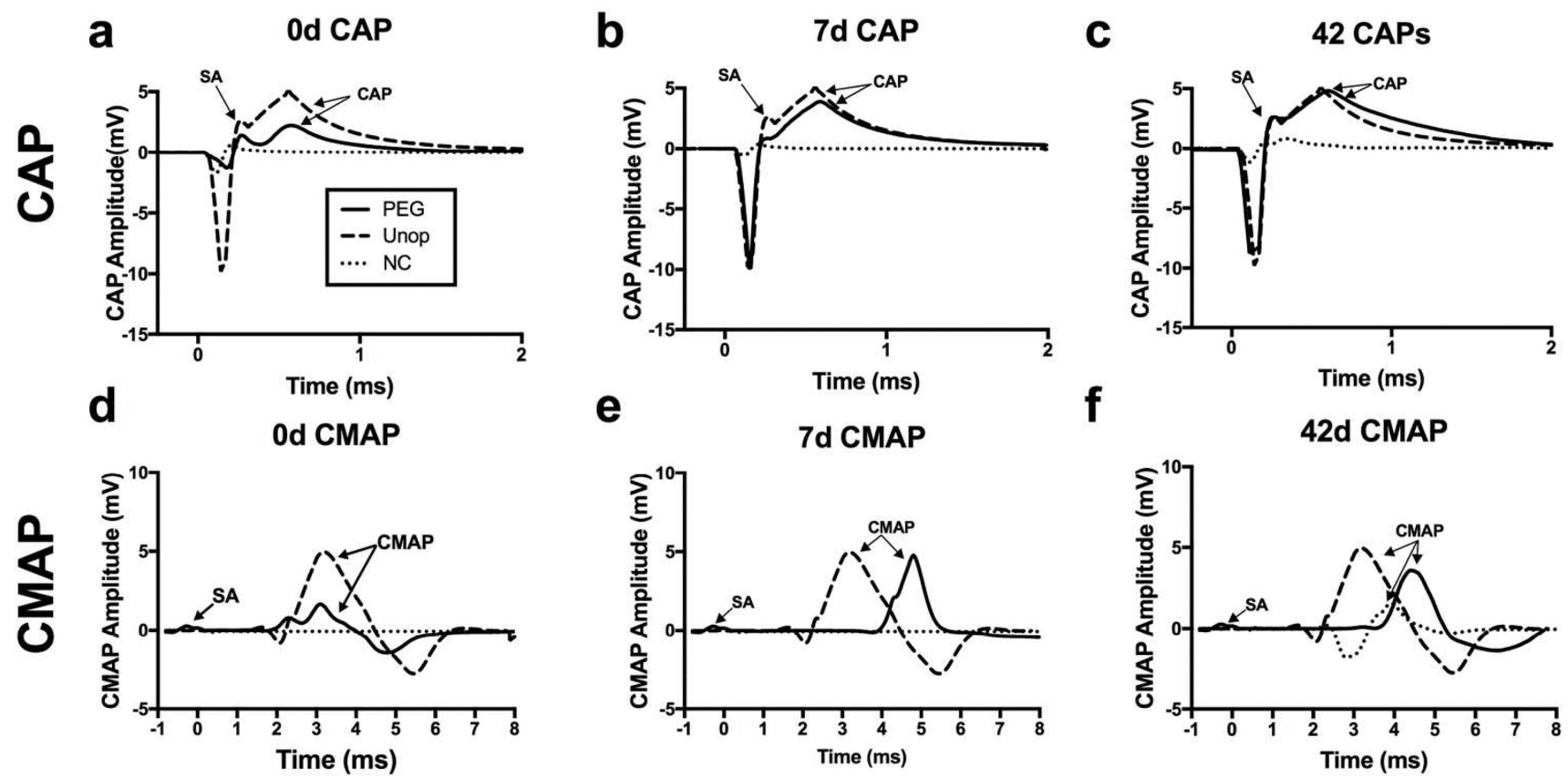

Figure 1.

Electrophysiological restoration after PEG-fusion of allografts for CAPs $(\mathrm{a}-\mathrm{c})$ and CMAPs (d-f). Panels a,d: Stimulus artifact (SA) for unoperated, intact nerve (Unop) before lesioning shown as dotted line in all panels. Panels b,c and e,f: The CAP or CMAP recorded from the originally-intact nerve is plotted to give a "baseline reference response" to compare visually with CAPs and CMAPs recorded from PEG-fused (PEG) and Negative Control (NC) nerves at $7 \mathrm{~d}$ and $42 \mathrm{~d} \mathrm{PO}$, respectively. NC nerves (dashed line in all panels) are microsutured and all solutions applied except PEG. PEG-fused allografts plotted as solid line in panels a-f. The same abbreviations are used in other figures. 

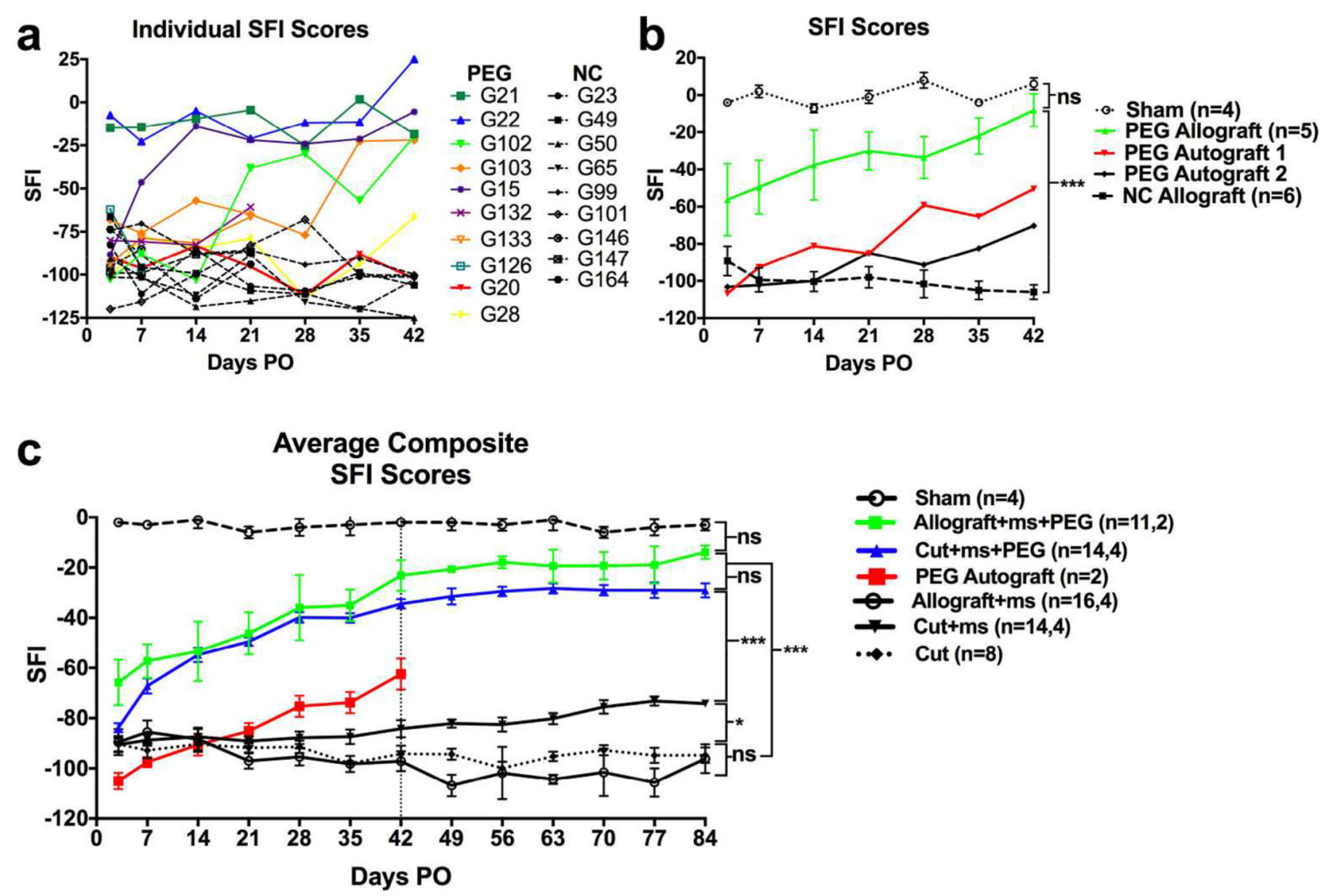

Figure 2.

SFI Behavioral assessments of functional recovery. (a) SFI scores from $3 d$ to $42 d$ PO of individual animals identified in the key receiving a PEG-fused (PEG) or Negative Control (NC) Allograft (See Table 2). Note that some animals are sampled for morphometric analyses at $7 \mathrm{~d}$ and $42 \mathrm{~d}$ PO. (b) Average \pm SE of SFI scores from $3 \mathrm{~d}$ to $42 \mathrm{~d}$ PO for sham operated animals or animals receiving a PEG-fused or Negative Control allograft or individual animals receiving PEG-fused autografts. (c) Composite plot of average \pm SE for current SFI results for rats having various surgical procedures on their sciatic nerve listed in the key that used the same PEG-fusion protocols described in this and the preceding paper (Mikesh et al., 2018). The two $\mathrm{n}$ values in the key for each curve gives the number of animals sampled at $3 \mathrm{~d}-42 \mathrm{~d}$ PO and the number animals that continued to be sampled at $49 \mathrm{~d}$ - 84d PO, respectively. Vertical bar marks 42d PO for which vertical bars and asterisks at right of graphs bars show $\mathrm{p}$ value comparisons $*, * *, * * *=\mathrm{p}<0.05,0.01$, and $<0.001$, respectively; $\mathrm{ns}=$ not significant $(\mathrm{p}>0.05)$. 

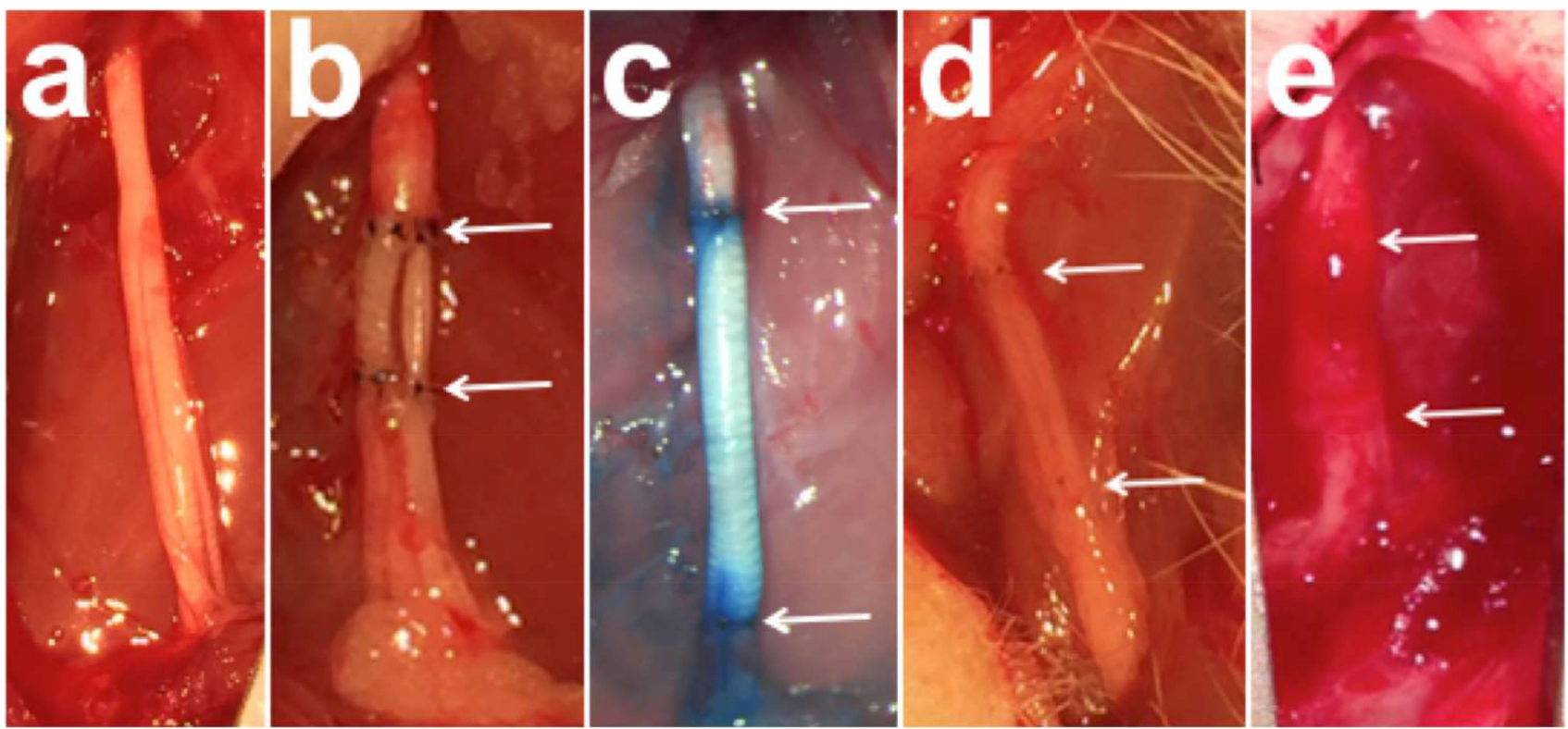

Figure 3.

Gross morphology of rat sciatic nerves. Images taken using a surgical operating microscope of Unoperated (a), PEG-fused autograft (b) and PEG-fused allograft (c) sciatic nerves immediately after surgical repair. Gross morphological images of PEG-fused (d) and negative control (e) allografts $42 \mathrm{~d}$ PO after surgical repair. Arrows indicate proximal and distal neurorrhaphy sites of grafted nerve tissue. 

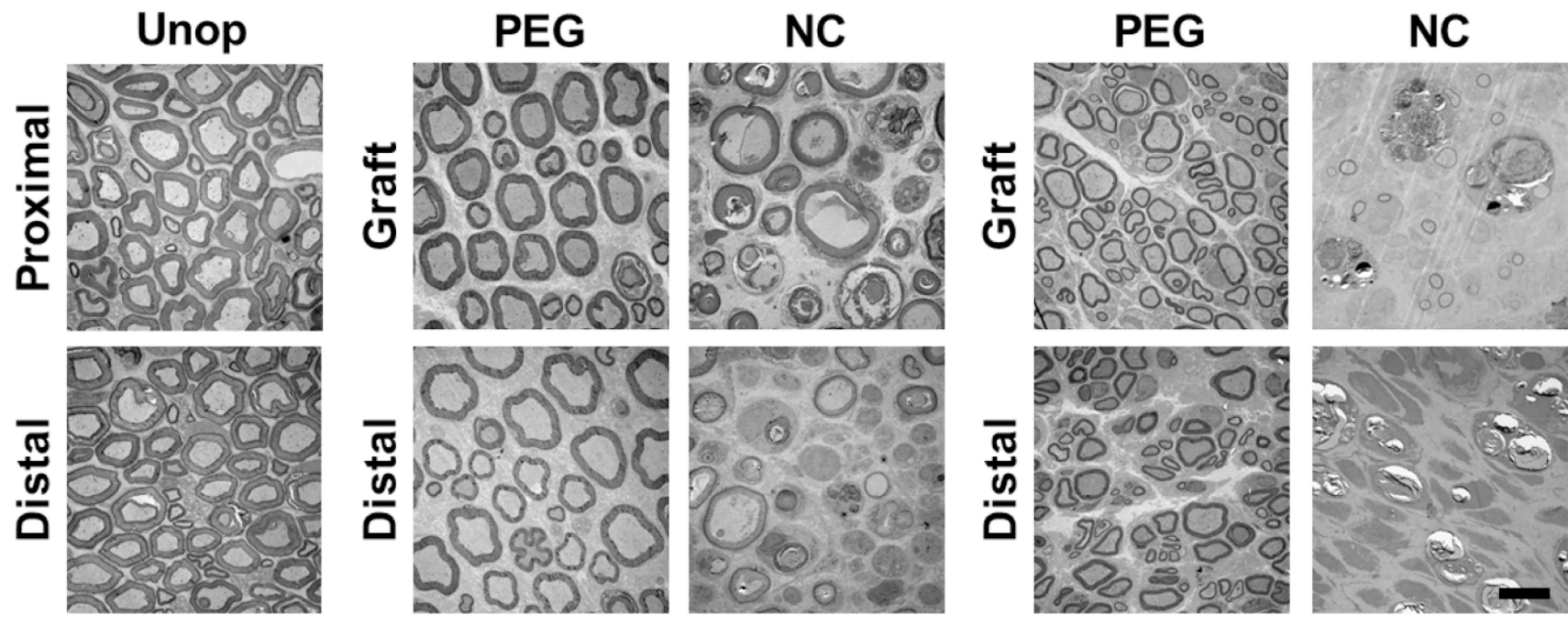

Od 7d PO

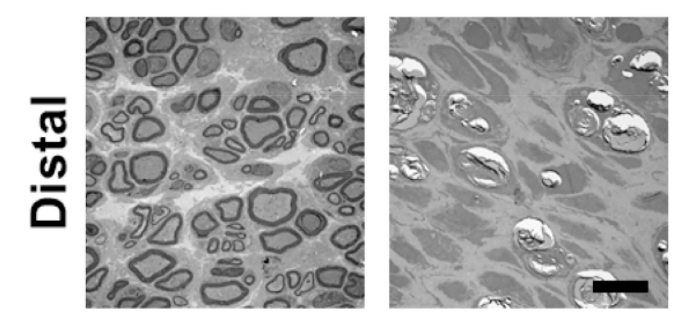

Figure 4.

TEM cross sections of Unoperated (intact) sciatic axons in the proximal and distal thigh of rats and rats and in the donor allograft and distal host nerve in thigh of rats with an allograft that spans a 5-10mm gap. The allograft was PEG-fused at both ends (PEG) or was only microsutured at both ends in Negative Control (NC) rats for which the sciatic nerves were examined at $7 \mathrm{~d}$ or $21 \mathrm{~d}$ PO. Scale bar $=10 \mu \mathrm{m}$. 

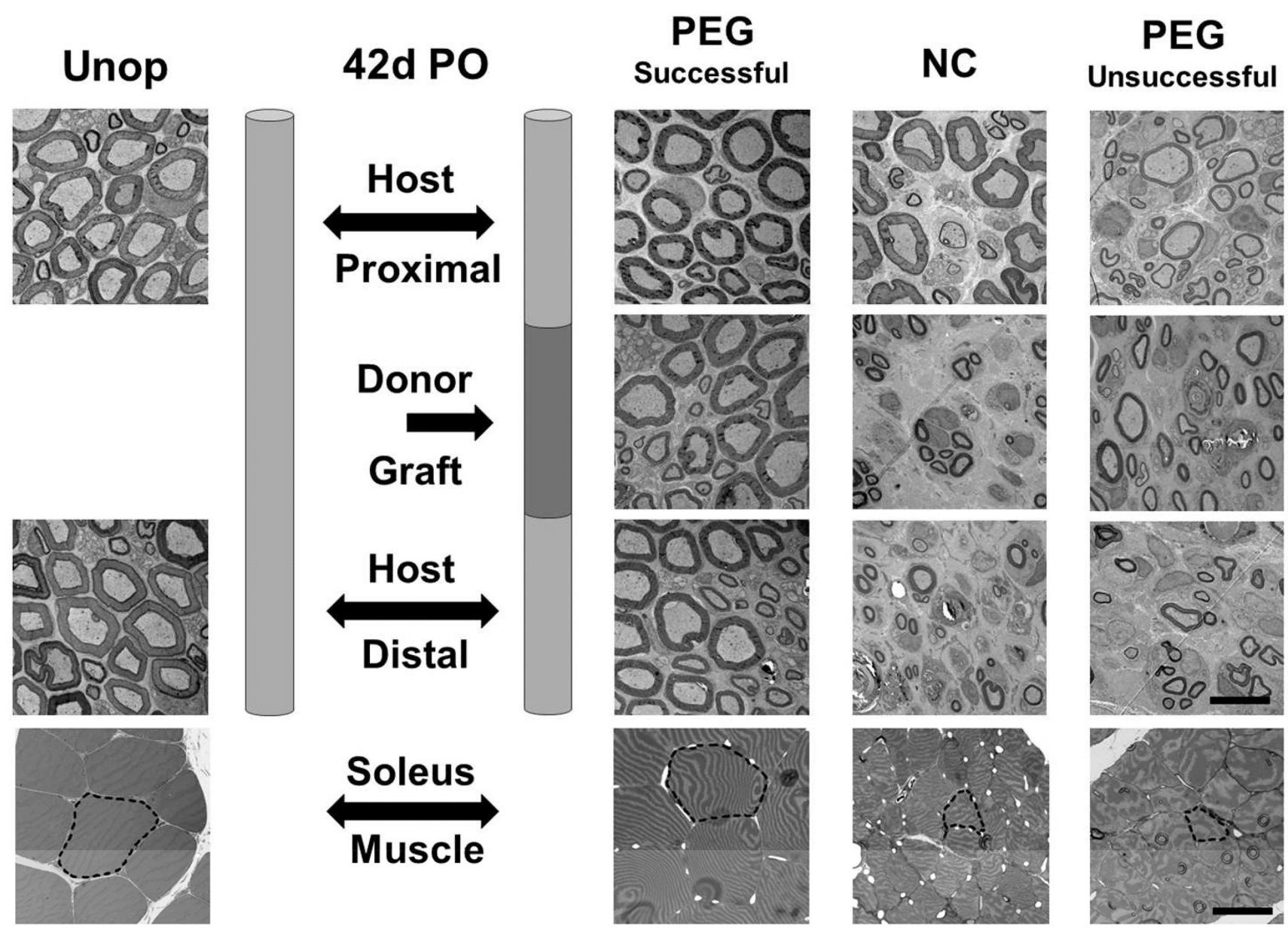

Figure 5.

Representative TEM cross sections from Unoperated sciatic nerves or from rats with an allograft that bridges a $5-10 \mathrm{~mm}$ gap. The allograft was microsutured and PEG-fused or was only microsutured at both cut ends in Negative Control rats and the sciatic nerve examined at $42 \mathrm{~d}$ PO proximal to the graft, within the graft, and distal to the graft. Note that PEG-Fused allografts in the proximal, graft and distal segments show some increases in interspace and have slightly smaller myelinated axons, but their morphology closely resembles Unoperated Controls. Negative Control allografts have a few smaller $(<4 \mu \mathrm{m})$ diameter axons in the allograft and even fewer more-distally. PEG-fused muscle fibers look very similar to unoperated fibers, but are atrophied in the Negative Control animal. Scale bars $=10 \mu \mathrm{m}$ for axons, $50 \mu \mathrm{m}$ for muscle fibers. 

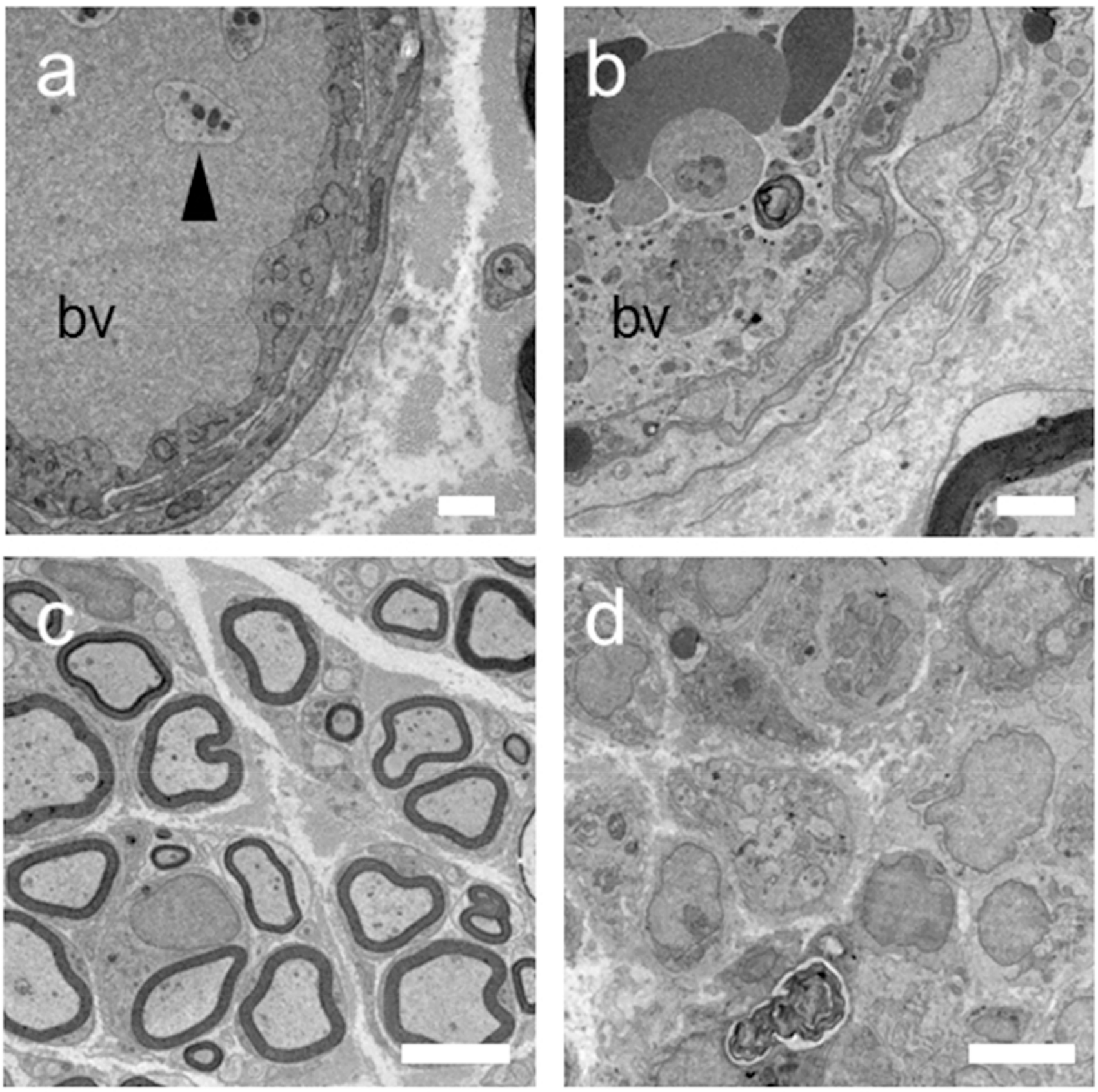

Figure 6.

Higher-power TEM of PEG fused (a,c) and Negative Control (b,d) allografts at $7 d$ PO (a,b)

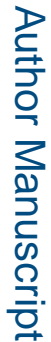
and $21 \mathrm{~d} P O(c, d) . b v=$ blood vessel, arrowhead $=$ non-RBC cell. Scales: $a=1 \mu \mathrm{m}, \mathrm{b}=2 \mu \mathrm{m}$, $c \& d=5 \mu \mathrm{m}$. 

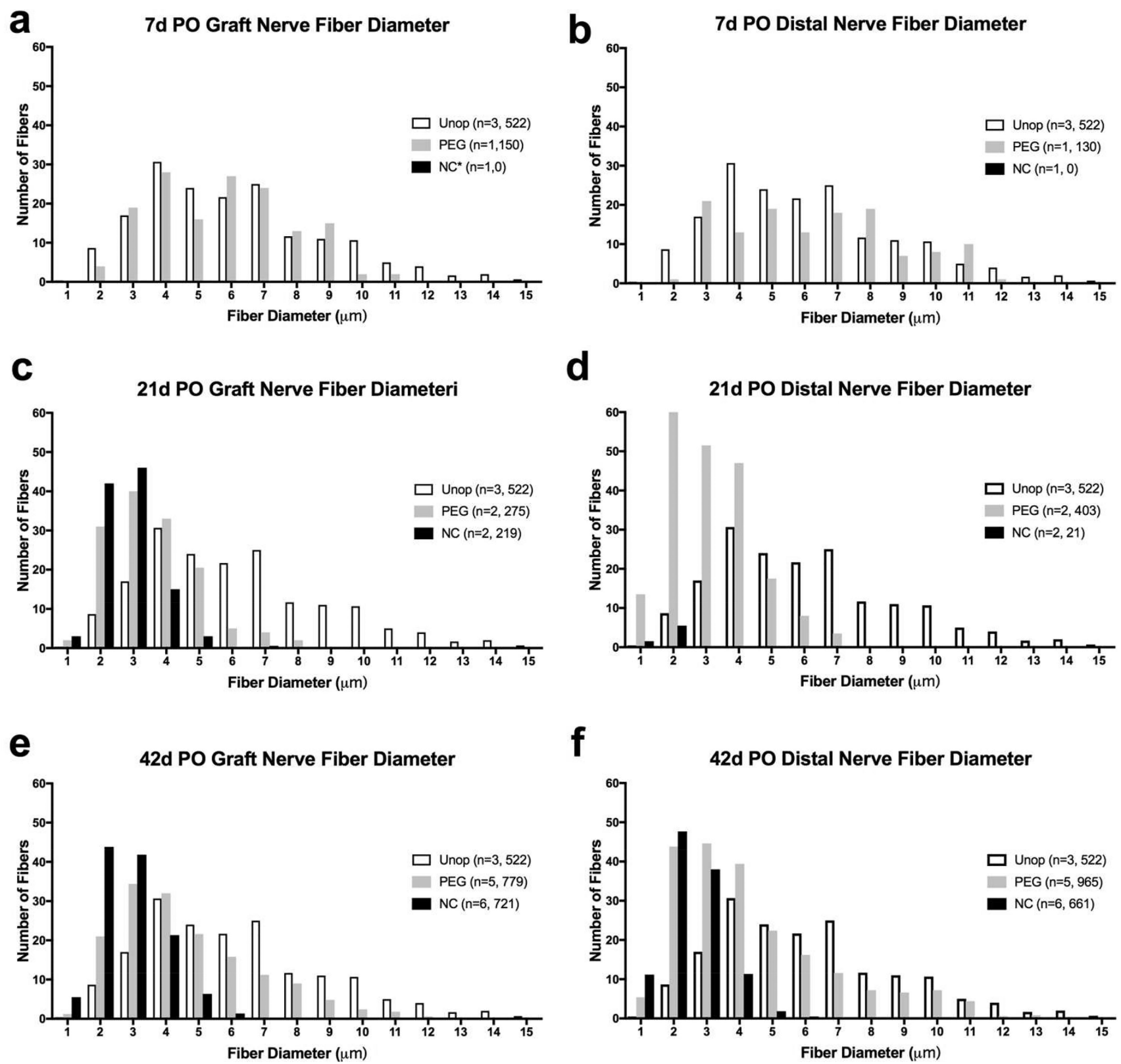

Figure 7.

Histograms of nerve fiber diameters versus average number of fibers per $10,000 \mu \mathrm{m} 2$ for a sciatic nerve. Bin width is $1 \mu \mathrm{m}(1-1.99 \mu \mathrm{m}, 2-2.99 \mu \mathrm{m}$, etc.) for intact Unoperated (Unop) nerve and for PEG-fused (PEG), and Negative Control (NC) graft segments (a, b, c) or distal nerve stumps $(\mathrm{d}, \mathrm{e}, \mathrm{f})$ at $7 \mathrm{~d}, 21 \mathrm{~d}$, and $42 \mathrm{~d}$ PO. $\mathrm{n}=$ total number of sciatic nerves, total number of nerve fibers measured. $\mathrm{NC}^{*}=$ no intact axons present at $7 \mathrm{~d} \mathrm{PO}$ in Negative Control. Note that Unoperated fibers range from 1-15 $\mu \mathrm{m}$, and PEG-fused fibers maintain a similar range at $7 \mathrm{~d}, 21 \mathrm{~d}$ and $42 \mathrm{~d}$ in the graft and distal segments. Note the absence of NC axons at $7 \mathrm{~d}(\mathrm{~b})$, followed by increasing number of smaller-diameter fibers $21 \mathrm{~d}(\mathrm{~d})$ to $42 \mathrm{~d}(\mathrm{~g})$ PO. 
a

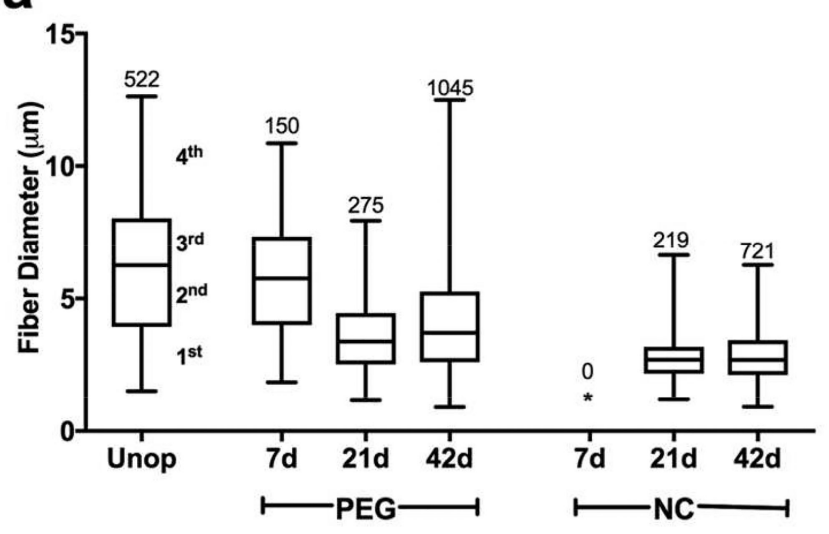

b

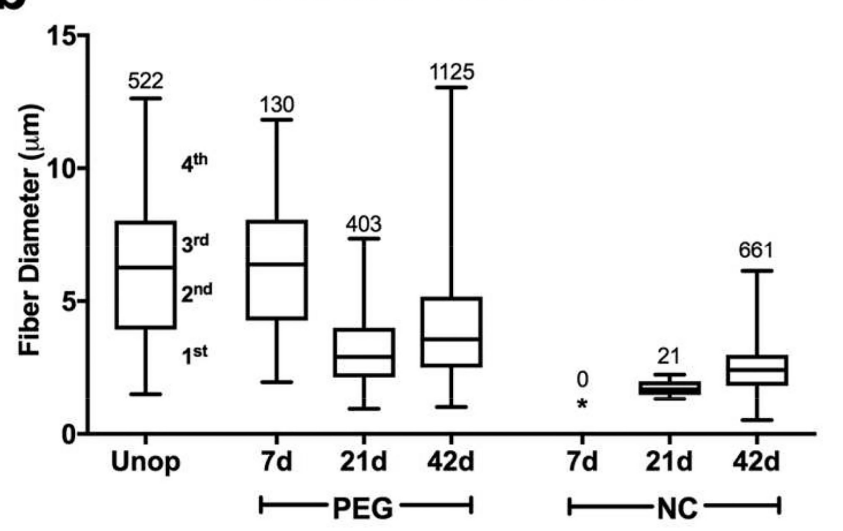

Figure 8.

Box plots showing range of nerve fiber diameters $(\mu \mathrm{m})$ for each quartile. Plots shown for range of fiber diameters in Unoperated sciatic nerves and in PEG-fused (PEG) and Negative Control (NC) sciatic nerves at 7d, 21d, and 42d PO for transected nerves in mid allograft (a) and $4 \mathrm{~mm}$ distal to the allograft in the distal host nerve (b). The line separating the 2 nd and 3rd quartiles shows the median diameter. * signifies no surviving axons. Numbers at the top of the highest quartile gives the total number of nerve fibers counted for that protocol (also see Table 2 for number of animals in each data set). 
a

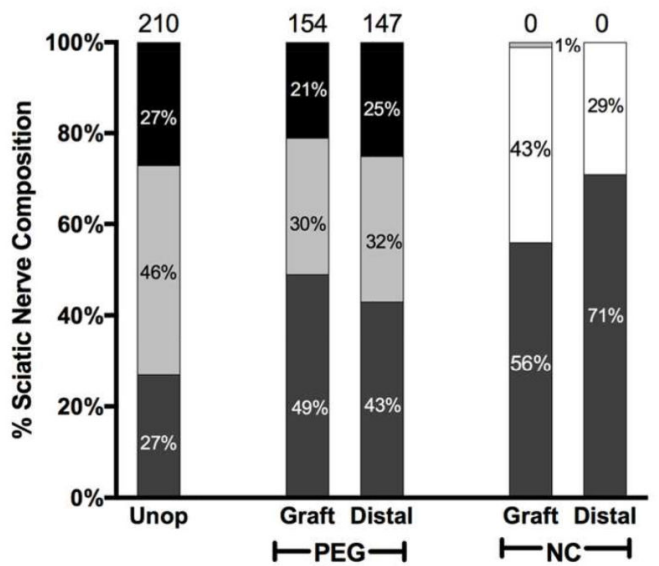

b 21d PO Nerve Composition

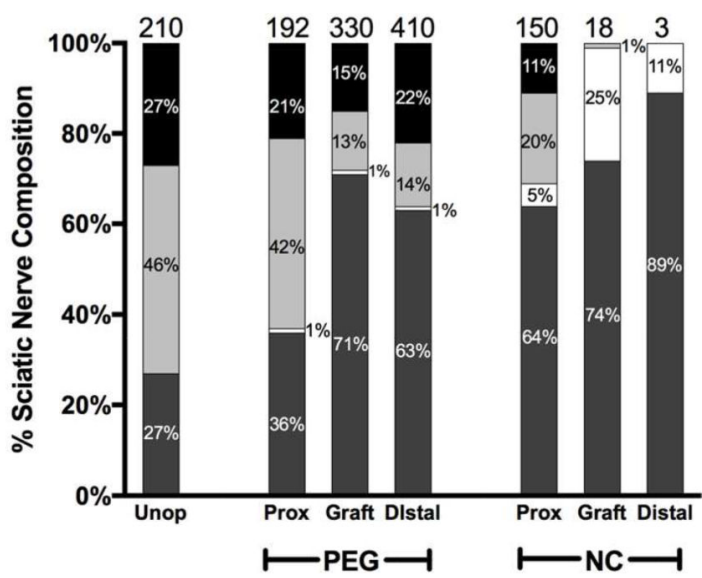

C

42d PO Nerve Composition

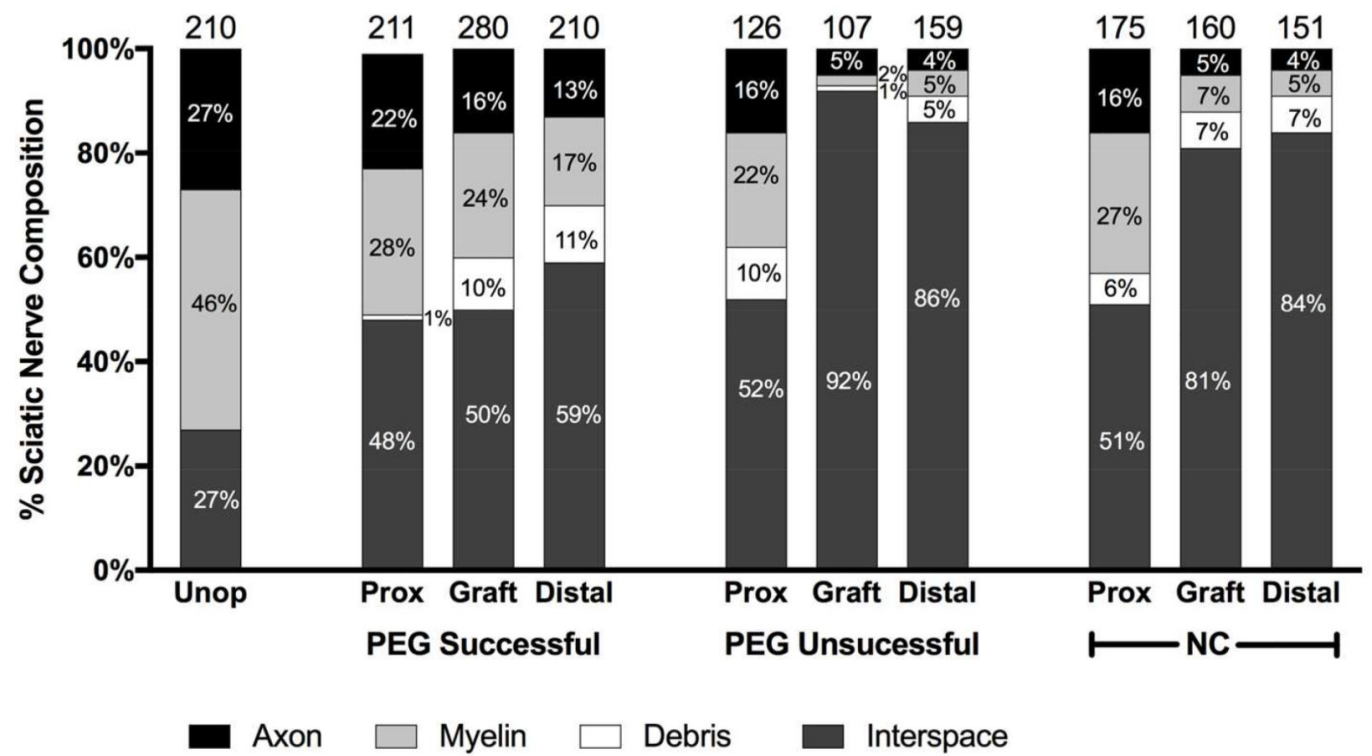

Figure 9.

Sciatic nerve composition: Sciatic nerves of Unoperated (Unop), PEG-fused, and Negative Control nerves were sampled at 7d (a), 21d (b), and 42d PO (c) in the proximal (P), graft $(G)$, and distal (D) segments. Bars represent the total area normalized to $10,000 \mu \mathrm{m} 2$. The relative percent of the total area taken up normal-appearing axons, myelin, and interspace and abnormal debris is represented by the partitions separating different bar shades of black, white or grey (see Key) and \% values within or beside each bar. The number of axons per $10,000 \mu \mathrm{m} 2$ for the segment is given at the top of each bar. 


\section{Unoperated}
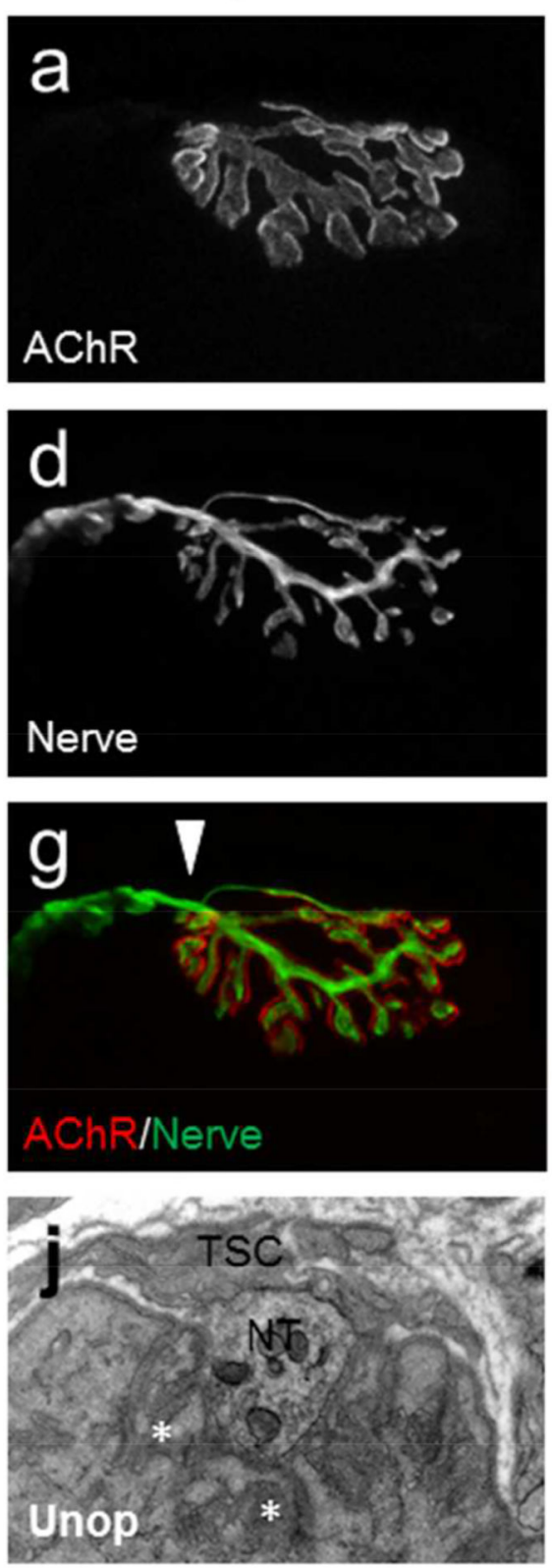

PEG-fused
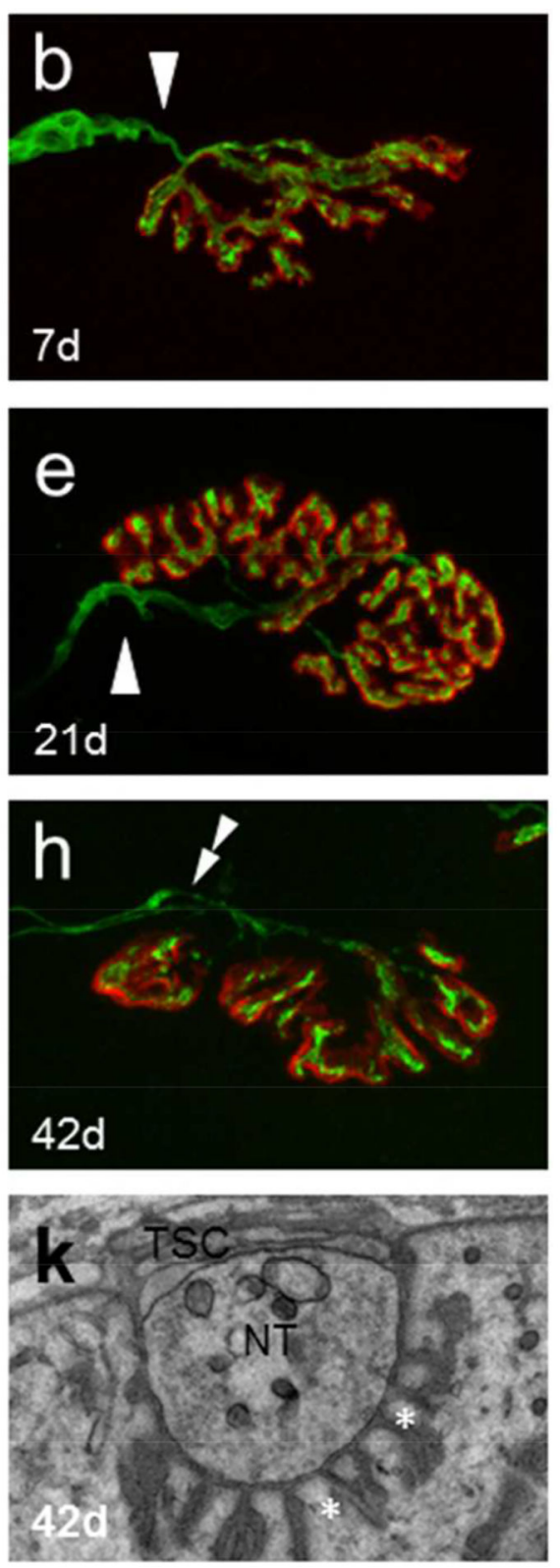

\section{Negative Control}
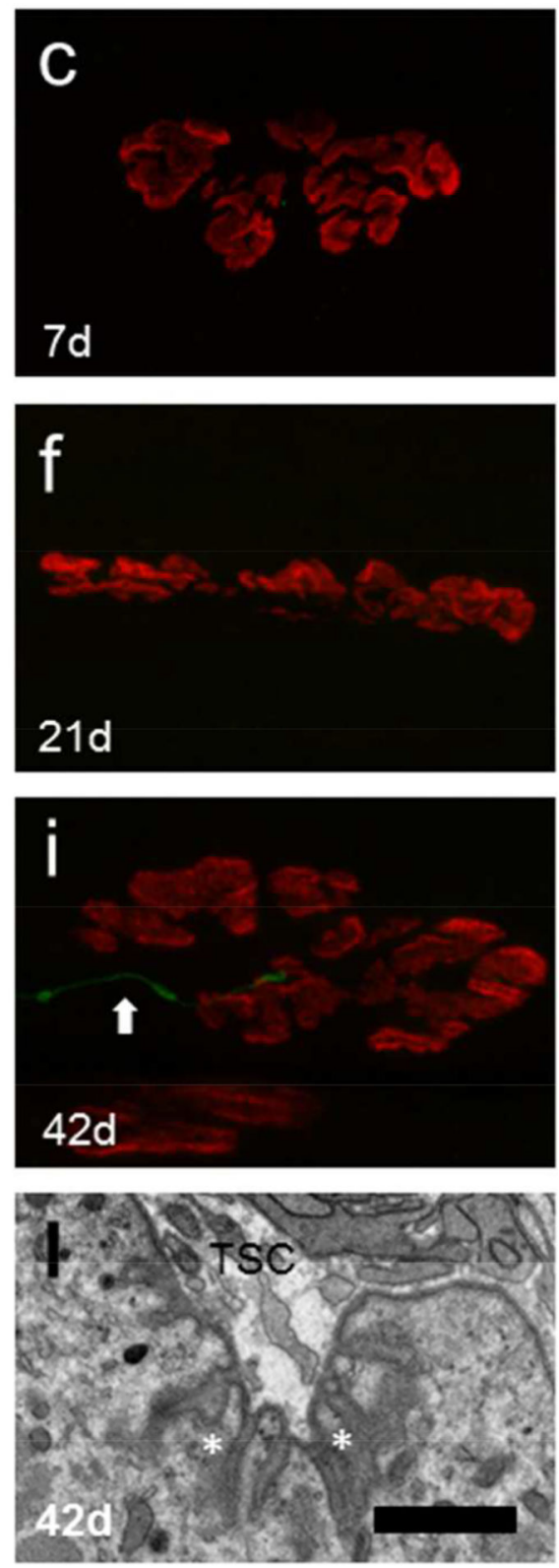

Figure 10.

Immunofluorescent confocal and electron micrographs of NMJs in soleus muscles distal to Unoperated (a,d,g,j), PEG-fused (b,e,h,k), and Negative Control (c,f,i,l) allograft repaired sciatic nerves $7 d$ (b,c), 21(e,f), and 42d PO (h,i,k,l) after ablation and repair. In normal NMJs, innervating axons (green) enter the synapse singly (arrowheads) and their nerve terminals mirror the shape of ACHRs (red). PEG-fused nerve terminals have normal ultrastructural configuration and occupy synaptic clefts $(\mathrm{k})$. Multiple innervation (double arrow, h) was common, as were retraction bulbs from innervated NMJs (not shown). At 42d days, Negative Control nerves did not closely appose AChRs (arrow, i) and many secondary 
folds were apposed by Schwann cell processes (1). Scale bars $=20 \mu \mathrm{m}$ for confocal $(\mathrm{a}-\mathrm{i}), 1 \mu \mathrm{m}$ for TEM $(j-1)$. 


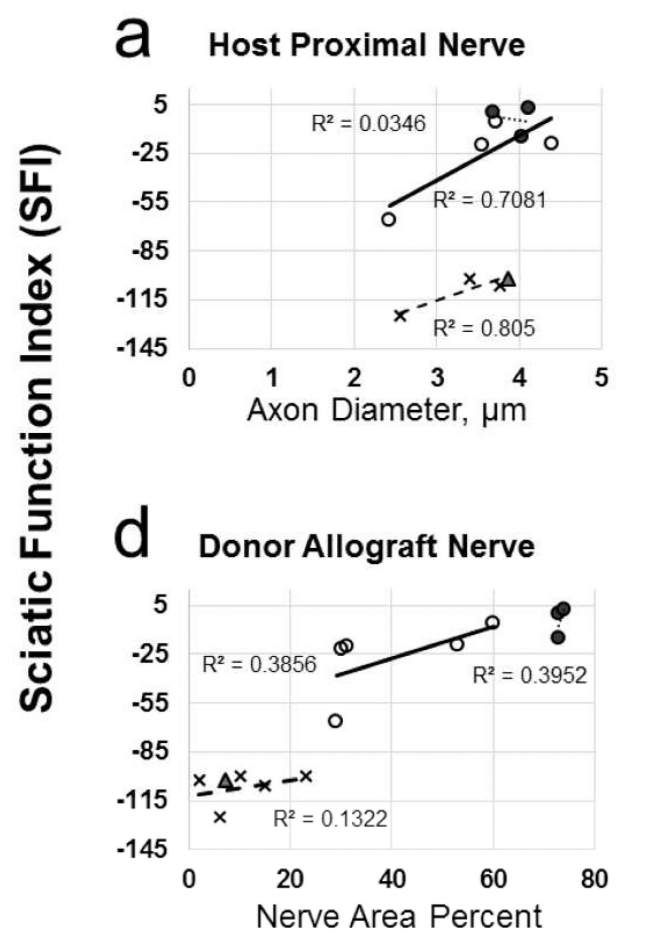

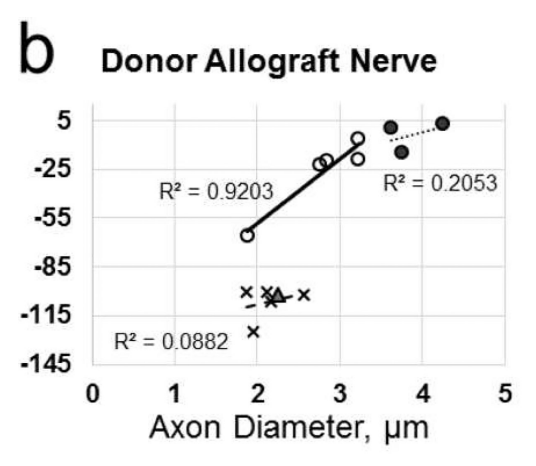
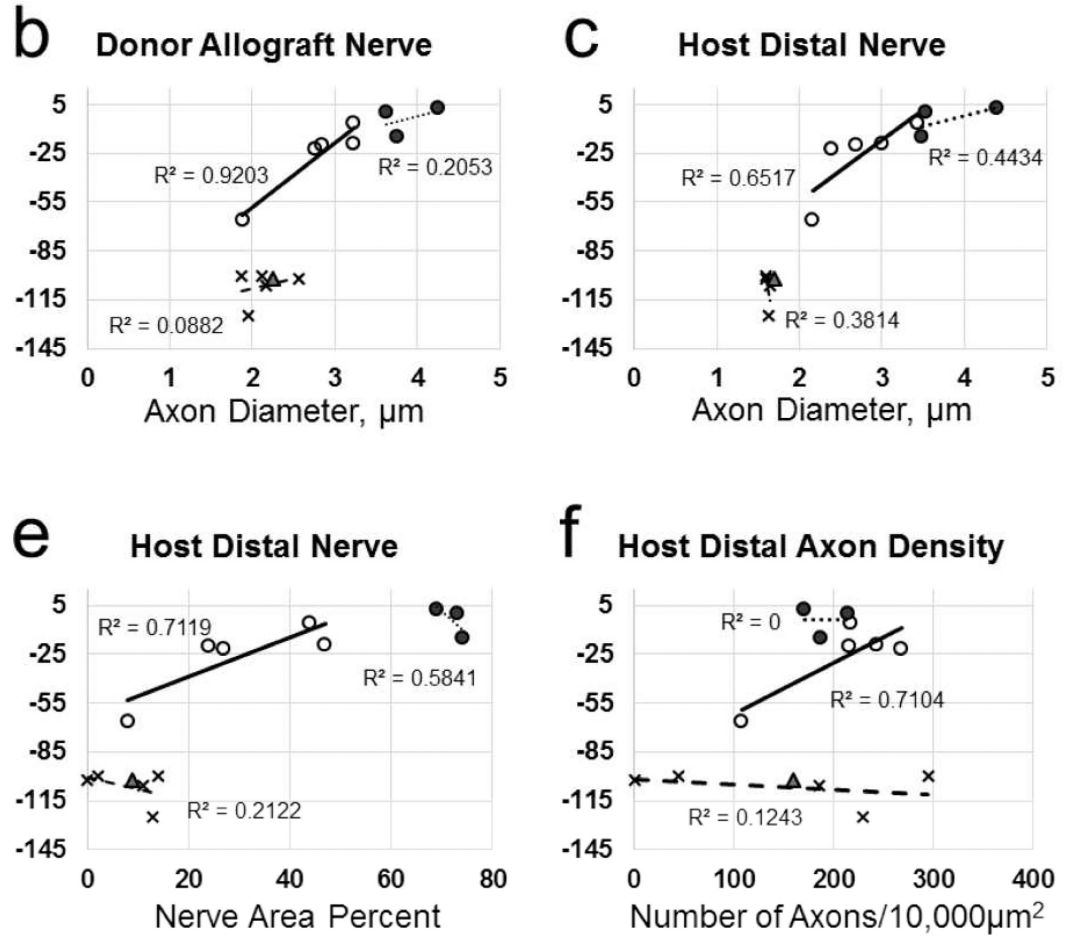

\section{f Host Distal Axon Density}

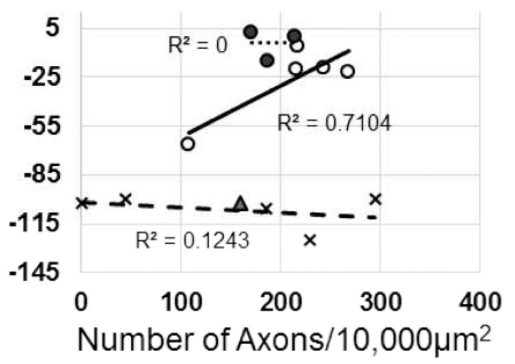

Figure 11.

SFI scores assessing function/behavior versus various morphometric measures for PEGfused allografts at $42 \mathrm{~d}$ PO. Individual Unoperated nerves (see Table 2) are plotted as filled circles with dotted linear best-fit regression line. Individual successfully PEG-fused nerves are plotted as open circles with solid regression line. The first $p$ value in the test is given for this line and these points, the second $p$ value is for all PEG-fused nerves, i.e., includes the Unsuccessful PEG-fused nerve (animal G20, Table 2) plotted as a grey-filled triangle.

Individual Negative Control nerves plotted as an $\times$ with dashed regression line. $\mathrm{P}$ values for each SFI correlation in each panel are as follows where PEG consists of animals G15, G21, G102, G103, and G28 and all PEG also includes animal G20 (unsuccessful PEG fusion). 


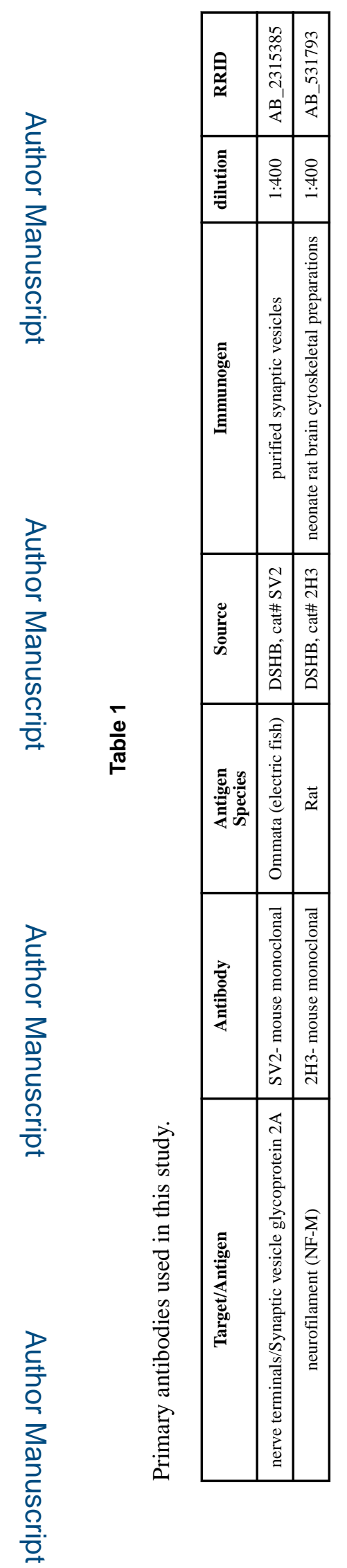

J Neurosci Res. Author manuscript; available in PMC 2019 January 01. 


\section{Table 2}

For each protocol (Unoperated: Unop, PEG-fused: PEG and Negative Control: NC), and each PO time (7d, 21d, 42d), Table 2 lists: SFI scores, axon diameter $(\mu \mathrm{m})$, g ratio (axon diameter/fiber diameter), axon density (per 10,000 $\mu \mathrm{m} 2$ ), average fiber diameter and the average of the ten largest fibers (MAX10), muscle fiber cross sectional area (MFA), and \% of muscle fibers having innervated NMJs (MFI). Axon diameters, $g$ ratios, and muscle fiber areas are given in boldfaced font as their average $\pm \mathrm{SD}$ when $\mathrm{n} \geq 2$. Data for individual animals \pm $\mathrm{SD}$ are in normal grey font. The average \pm value for all animals in a given protocol at a given PO time are in boldfaced font. Note that PEG fused nerves have intact axons in all regions at all PO times. Compared to Unoperated nerves, nerve fiber diameter is slightly reduced in the distal segments of PEG-fused nerves, but absent (7d and/or 21d PO) or greatly reduced in Negative Controls nerves. PEG-fusion maintains functional NMJs and reduces or prevents muscle fiber atrophy seen in Negative Controls and Unsuccessful PEG-fusion.

\begin{tabular}{|c|c|c|c|c|c|c|c|c|c|c|c|c|c|c|c|c|}
\hline \multirow[t]{2}{*}{ Days PO } & \multirow{2}{*}{$\begin{array}{l}\text { Animal, } \\
\text { group }\end{array}$} & \multirow[t]{2}{*}{ SFI } & \multicolumn{3}{|c|}{ Avg axon diameter $(\mu \mathrm{m})$} & \multirow[b]{2}{*}{ prox } & \multirow{2}{*}{ Avg g ratio } & \multirow[b]{2}{*}{ distal } & \multicolumn{3}{|c|}{ Avg Nerve Fiber $\left(\mathrm{MAX}_{10}\right)$} & \multicolumn{3}{|c|}{ Axons/10,000 $\mu \mathrm{m} 2$} & \multirow{2}{*}{$\begin{array}{l}\text { MFA } \\
\left(\mu m^{2}\right)\end{array}$} & \multirow{2}{*}{$\begin{array}{l}\text { MFI } \\
(\%)\end{array}$} \\
\hline & & & prox & graft & distal & & & & prox & graft & distal & prox & graft & distal & & \\
\hline \multicolumn{17}{|l|}{ Unoperated } \\
\hline & $\mathrm{U} 2$ & 3 & $4.11 \pm 1.70$ & {$[4.25]$} & $4.40 \pm 1.96$ & $0.62 \pm 0.05$ & {$[0.62]$} & $0.62 \pm 0.06$ & $6.7(11.2)$ & $[6.8(12.0)]]$ & $7.0(11.9)$ & 201 & [186] & 171 & & $100 \%^{\circ}$ \\
\hline & $\mathrm{U1}$ & 0 & $3.69 \pm 1.64$ & [3.61] & $3.54 \pm 1.50$ & $0.63 \pm 0.05$ & {$[0.62]$} & $0.61 \pm 0.05$ & $5.9(10.7)$ & {$[5.8(10.8)]$} & $5.9(10.3)$ & 241 & [228] & 215 & $2690 \pm 700$ & $100 \%^{*}$ \\
\hline & 43 & -15 & $4.03 \pm 1.86$ & {$[3.77]$} & $3.49 \pm 1.16$ & $0.60 \pm 0.06$ & {$[0.61]$} & $0.61 \pm 0.06$ & $6.6(11.8)$ & {$[6.2(11.8)]$} & $5.7(9.2)$ & 187 & {$[187]$} & 187 & $2730 \pm 720$ & $100 \%^{*}$ \\
\hline Unop Avg & $n=3$ & -4 & $3.95 \pm 1.74$ & [3.88] & $3.82 \pm 1.64$ & $0.62 \pm 0.06$ & {$[0.62]$} & $0.61 \pm 0.06$ & $6.4(12.0)$ & {$[6.3(11.5)]$} & $6.2(11.9)$ & 210 & [201] & 191 & $2710 \pm 710$ & $100 \%^{*}$ \\
\hline \multirow[t]{3}{*}{$7 \mathrm{dPO}$} & & & & & & & & & & & & & & & & \\
\hline & G126 PEG & -96 & & $3.88 \pm 1.41$ & $4.44 \pm 1.74$ & & $0.67 \pm 0.05$ & $0.69 \pm 0.03$ & & $5.8(9.5)$ & $6.4(11.0)$ & & 154 & 147 & & $50 \%$ \\
\hline & G146 NC & -85 & & 0 & 0 & & 0 & 0 & & 0 & 0 & & 0 & 0 & & $0 \%$ \\
\hline \multirow[t]{3}{*}{ 21d PO } & & & & & & & & & & & & & & & & \\
\hline & G132 PEG & -61 & & $2.55 \pm 0.95$ & $1.89 \pm 0.95$ & & $0.70 \pm 0.05$ & $0.60 \pm 0.09$ & & $3.6(6.0)$ & $3.1(6.2)$ & & 284 & 485 & & $84 \%$ \\
\hline & G133 PEG & -66 & $3.56 \pm 1.75$ & $2.67 \pm 1.20$ & $2.15 \pm 1.01$ & $0.62 \pm 0.08$ & $0.72 \pm 0.06$ & $0.64 \pm 0.07$ & $5.9(11.6)$ & $3.6(7.2)$ & $3.3(6.6)$ & 192 & 330 & 410 & & $97 \%$ \\
\hline \multirow{3}{*}{ 21d PEG Avg } & $\mathrm{n}=2$ & -64 & $3.56 \pm 1.75$ & $2.62 \pm 1.11$ & $2.01 \pm 0.99$ & $0.62 \pm 0.08$ & $0.72 \pm 0.06$ & $0.62 \pm 0.08$ & $5.9(11.6)$ & $3.6(7.4)$ & $3.2(6.9)$ & 192 & 307 & 448 & & $92 \%$ \\
\hline & G147 NC & -87 & $2.70 \pm 1.33$ & $2.31 \pm 0.81$ & 0 & $0.73 \pm 0.10$ & $0.80 \pm 0.07$ & 0 & $3.7(9.2)$ & $2.9(4.9)$ & 0 & 150 & 4 & 0 & & $0 \%$ \\
\hline & G164 NC & -93 & & $2.09 \pm 0.51$ & $1.35 \pm 0.22$ & & $0.81 \pm 0.05$ & $0.78 \pm 0.07$ & & $2.6(3.4)$ & $1.7(1.9)$ & & 31 & 6 & $620 \pm 210$ & \\
\hline 21d NC Avg & $n=2$ & -90 & $2.70 \pm 1.33$ & $2.24 \pm 0.74$ & $1.35 \pm 0.22$ & $0.73 \pm 0.10$ & $0.81 \pm 0.06$ & $0.78 \pm 0.07$ & $3.7(9.2)$ & $2.8(4.9)$ & $1.7(1.9)$ & 150 & 18 & 3 & $620 \pm 210$ & $0 \%$ \\
\hline \multirow{7}{*}{\begin{tabular}{|l}
$42 \mathrm{~d} \mathrm{PO}$ \\
\end{tabular}} & & & & & & & & & & & & & & & & \\
\hline & G22 PEG & 25 & & & & & & & & & & & & & $2880 \pm 670$ & \\
\hline & G15 PEG & -6 & $3.72 \pm 1.61$ & $3.22 \pm 1.77$ & $3.43 \pm 2.00$ & $0.66 \pm 0.07$ & $0.64 \pm 0.08$ & $0.66 \pm 0.07$ & $5.7(10.7)$ & $5.0(11.2)$ & $5.7(10.6)$ & 233 & 232 & 217 & $3040 \pm 1010$ & \\
\hline & G21 PEG & -19 & $4.39 \pm 1.93$ & $3.23 \pm 1.63$ & $3.01 \pm 1.96$ & $0.03 \pm 0.06$ & $0.64 \pm 0.06$ & $0.66 \pm 0.07$ & $6.8(12.2)$ & $5.0(10.0)$ & $4.5(11.9)$ & 175 & 262 & 243 & $2750 \pm 520$ & \\
\hline & G102 PEG & -20 & $3.55 \pm 1.61$ & $2.85 \pm 0.97$ & $2.69 \pm 1.16$ & $0.64 \pm 0.10$ & $0.67 \pm 0.08$ & $0.69 \pm 0.09$ & $5.5(10.5)$ & $4.2(6.4)$ & $3.8(6.8)$ & 187 & 256 & 216 & & $100 \%$ \\
\hline & G103 PEG & -22 & & $2.77 \pm 1.15$ & $2.39 \pm 1.28$ & & $0.64 \pm 0.07$ & $0.66 \pm 0.08$ & & $4.3(8.5)$ & $3.5(7.1)$ & & 304 & 268 & & $98 \%$ \\
\hline & G28 PEG** & -66 & $2.43 \pm 1.47$ & $1.88 \pm 0.76$ & $2.16 \pm 0.87$ & $0.69 \pm 0.09$ & $0.65 \pm 0.09$ & $0.69 \pm 0.07$ & $3.4(7.7)$ & $2.9(5.6)$ & $3.1(5.6)$ & 248 & 345 & 108 & $920 \pm 310$ & \\
\hline 42d PEG Avg & $n=5$ & -8 & $3.33 \pm 1.71$ & $2.87 \pm 1.43$ & $2.96 \pm 1.77$ & $0.64 \pm 0.07$ & $0.65 \pm 0.07$ & $0.67 \pm 0.08$ & $6.0(12.3)$ & $4.6(11.4)$ & $4.4(11.9)$ & 198 & 264 & 236 & $2890 \pm 780$ & $99 \%$ \\
\hline \multirow[t]{7}{*}{ 42d PEG Avg } & $n=6$ & -18 & $3.46 \pm 1.78$ & $2.82 \pm 1.48$ & $2.85 \pm 1.71$ & $0.66 \pm 0.08$ & $0.65 \pm 0.07$ & $0.67 \pm 0.08$ & $5.3(12.4)$ & $4.2(11.4)$ & $4.2(11.9)$ & 211 & 280 & 210 & $2360 \pm 1100$ & $99 \%$ \\
\hline & G99 NC & -100 & & $1.87 \pm 0.86$ & $1.59 \pm 0.64$ & & $0.65 \pm 0.10$ & $0.64 \pm 0.09$ & & $2.8(5.4)$ & $2.5(4.3)$ & & 358 & 45 & & $9 \%$ \\
\hline & G101 NC & -100 & & $2.12 \pm 0.88$ & $1.51 \pm 0.57$ & & $0.77 \pm 0.08$ & $0.65 \pm 0.14$ & & $2.7(5.1)$ & $2.3(4.1)$ & & 143 & 295 & & $8 \%$ \\
\hline & G23 NC & -101 & & & & & & & & & & & & & $730 \pm 150$ & \\
\hline & G65 NC & -102 & $340+149$ & $257+0.63$ & 159 & $0.65+0.08$ & $0.88+0.04$ & 0.5 & $54(10.5)$ & $29(37)$ & 239 & 159 & 32 & 1 & $790+230$ & \\
\hline & G49 NC & -106 & $3.76 \pm 1.95$ & $2.16 \pm 0.77$ & $1.64 \pm 0.66$ & $0.62 \pm 0.08$ & $0.73 \pm 0.08$ & $0.64 \pm 0.09$ & $6.0(11.7)$ & $2.9(5.0)$ & $2.5(4.7)$ & 133 & 178 & 186 & $920 \pm 300$ & \\
\hline & G50 NC & -125 & $2.55 \pm 1.29$ & $1.96 \pm 0.76$ & $1.63 \pm 0.62$ & $0.63 \pm 0.08$ & $0.66 \pm 0.08$ & $0.60 \pm 0.08$ & $4.2(9.3)$ & $2.9(5.1)$ & $2.7(4.5)$ & 232 & 89 & 229 & $680 \pm 270$ & \\
\hline & $n=6$ & -106 & $\frac{3.27 \pm 1.29}{3.27 \pm 1.63}$ & $\frac{1.0 \pm 0.10}{2.07 \pm 0.83}$ & $\frac{1.03 \pm 9.02}{1.59 \pm 0.62}$ & $\begin{array}{l}0.0 .64 \pm 0.08 \\
0.00\end{array}$ & $\frac{.0050 .06}{0.72 \pm 0.10}$ & $\frac{.0 .635 .06}{0.63 \pm 0.10}$ & $\begin{array}{l}4.2(1.31 .8) \\
5.2(11)\end{array}$ & $\begin{array}{l}2.9(5.1) \\
2.8(5.7)\end{array}$ & $\begin{array}{l}2.9(1.5) \\
2.5(5.1)\end{array}$ & $\frac{152}{175}$ & 160 & 151 & $\frac{000 \pm 210}{790 \pm 260}$ & $9 \%$ \\
\hline \multirow{2}{*}{\begin{tabular}{|l} 
Unsuccessful \\
42d PO PEG \\
\end{tabular}} & & & & & & & & & & & & & & & & \\
\hline & G20 U-PEG & -102 & $3.86+1.97$ & $2.25 \pm 0.8$ & $1.70 \pm 0.61$ & $0.66 \pm 0.06$ & $0.86 \pm 0.05$ & $0.69 \pm 0.08$ & $5.8(12.4)$ & $2.6(4.7)$ & $2.4(4.2)$ & 126 & 107 & 159 & $990 \pm 290$ & \\
\hline
\end{tabular}




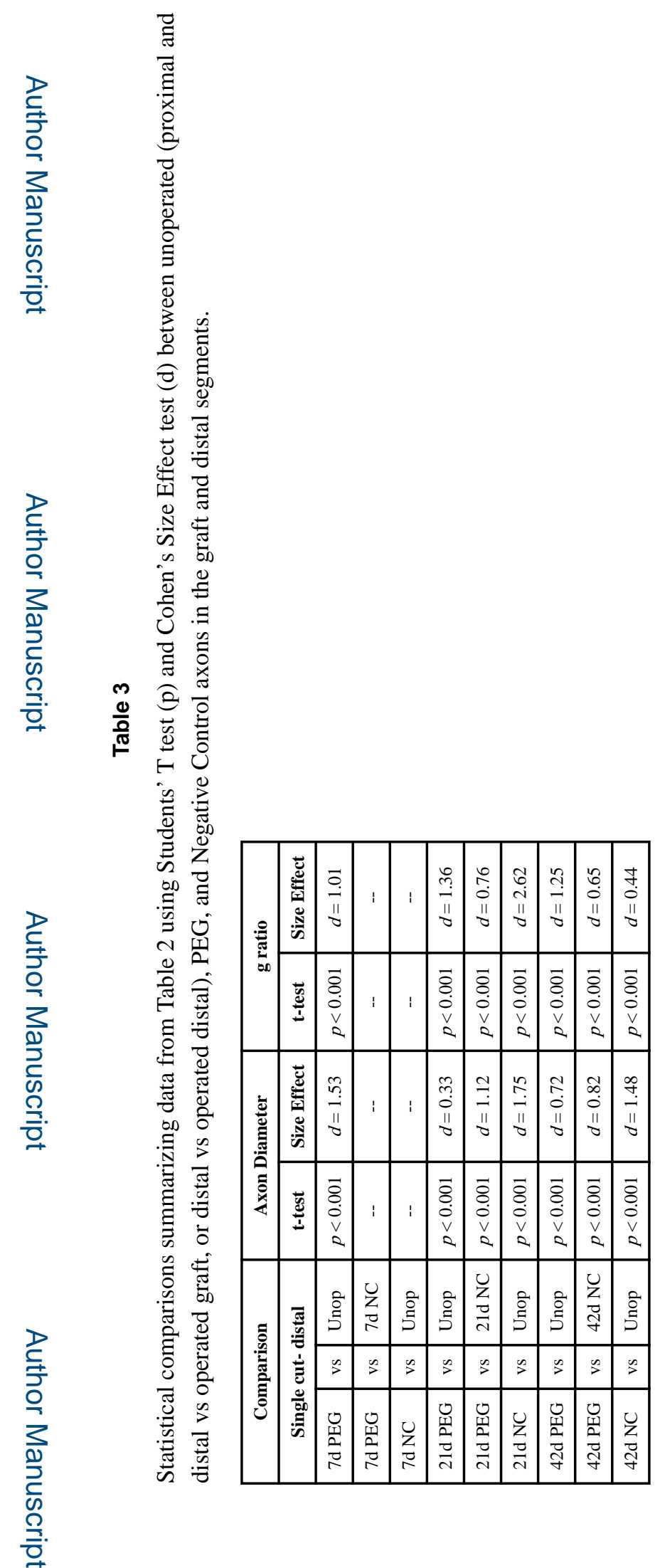

J Neurosci Res. Author manuscript; available in PMC 2019 January 01. 


\section{Table 4}

Summary of means for single cut and allograft axonal morphometric data. Table 4 lists allograft ablation data (black font) versus single cut data (red font) for sciatic nerve lesions in the mid-thigh for each protocol and PO times, as given in the 1st column.

\begin{tabular}{|c|c|c|c|c|c|c|c|c|c|c|c|c|}
\hline \multirow[t]{2}{*}{ Treatment } & \multirow[t]{2}{*}{ SFI } & \multicolumn{3}{|c|}{ Mean axon diameter $\left(\mu_{\mathrm{m}}\right)$} & \multicolumn{3}{|c|}{ Mean g ratio } & \multicolumn{3}{|c|}{ Axons $/ 10,000 \mu \mathrm{m} 2$} & \multirow{2}{*}{$\begin{array}{l}\text { MFA } \\
\left(\mu \mathrm{m}^{2}\right)\end{array}$} & \multirow{2}{*}{$\begin{array}{l}\text { MFI } \\
(\%)\end{array}$} \\
\hline & & prox & graft & distal & prox & graft & distal & prox & graft & distal & & \\
\hline \multicolumn{13}{|l|}{ Unoperated } \\
\hline Unoperated mean & -4 & 3.95 & [3.88] & 3.82 & 0.62 & {$[0.62]$} & 0.61 & 210 & [201] & 191 & 2710 & $100 \%$ \\
\hline \multicolumn{13}{|l|}{ 7-14d PO } \\
\hline PEG mean & $-96(-90)$ & & 3.88 & $4.44(2.69)$ & & 0.67 & $0.69(0.66)$ & & 154 & $147(196)$ & & $50 \%(80 \%)$ \\
\hline NC mean & $-85(-98)$ & & 0 & $0(0)$ & & 0 & $0(0)$ & & 0 & $0(0)$ & 1370 & $0 \%(0 \%)$ \\
\hline \multicolumn{13}{|l|}{ 21d PO } \\
\hline PEG mean & $-64(-70)$ & 3.56 & 2.62 & $2.01(3.24)$ & 0.62 & 0.71 & $0.62(0.71)$ & 192 & 307 & $448(150)$ & & $91 \%(78 \%)$ \\
\hline $\mathrm{NC}$ mean & $-90(-87)$ & 2.70 & 2.24 & $1.35(1.72)$ & 0.73 & 0.80 & $0.78(0.76)$ & 150 & 18 & $3(116)$ & 620 & $0 \%(0 \%)$ \\
\hline \multicolumn{13}{|l|}{$42 \mathrm{dPO}$} \\
\hline PEG mean & $-8(-29)$ & $3.33(3.82)$ & 2.87 & $2.96(2.77)$ & $0.64(0.64)$ & 0.65 & $0.67(0.70)$ & $198(190)$ & 264 & 236 (231) & 2890 (1780) & $99 \%(100 \%)$ \\
\hline NC mean & $-106(-85)$ & $3.27(3.37)$ & 2.07 & $1.59(1.90)$ & $0.64(0.66)$ & 0.72 & $0.63(0.65)$ & $175(199)$ & 160 & $151(237)$ & 790 (1440) & $9 \%$ \\
\hline
\end{tabular}

Abbreviations as defined for Table 2 of this paper and for Table 2 of Mikesh et al. (2018). 